June 2013

"Physical Activity and Policy Recommendations: a Social Multiplier Approach"

Catarina GOULAO and Emmanuel THIBAULT 


\title{
Physical Activity and Policy Recommendations:
}

\section{a Social Multiplier Approach*}

\author{
Catarina GOULÃO \\ Toulouse School of Economics (GREMAQ and INRA)

\section{Emmanuel THIBAULT}

Toulouse School of Economics (University of Perpignan, CDED and IDEI)

\begin{abstract}
We look at the effects of physical activity (PA) recommendation policies by considering a social multiplier model in which individuals differ in their concern for PA. The government can either observe this concern (and implement the First Best) or not (and implement a uniform policy). Whichever the type of policy implemented, while the welfare of individuals the most concerned with PA increases in the social multiplier, the welfare of those the least concerned may decrease in it. For a sufficiently high social multiplier, both government interventions improve the welfare of those most concerned with PA but worsen the welfare of the least concerned individuals if they are not too many. However, compared to the First Best, a uniform recommendation improves the welfare of those most concerned with PA more than it reduces the welfare of those least concerned.
\end{abstract}

Keywords: Physical Activity, Peer Effects, Long Term Care.

JEL codes: I 18, D 62, H 11.

\footnotetext{
${ }^{*}$ We are grateful to David Bardey and Pierre Pestieau for their comments and suggestions. We also thank participants at the "First workshop IDEI/SCOR/TSE of Long Term Care" (Toulouse, January 6, 2011), and at the "CESifo Workshop on the Economics of Long Term Care" (Venice, July 18-19, 2012) for their discussions. E. Thibault thanks the Chair Fondation du Risque/SCOR "Marché du risque et création de valeurs" for its financial support.
} 


\section{Introduction}

Using data for adults from 122 countries, Hallal et al (2012) recently revealed that, worldwide, $31.1 \%$ of adults are physically inactive, with proportions ranging from $17 \%$ in southeast Asia to around $43 \%$ in the Americas and the eastern Mediterranean. Inactivity rises with age, is higher in women than in men, and is increased in high-income countries. These constitute unfavorable statistics from a public health perspective since physical inactivity is, respectively, the fourth and fifth leading cause of death and disability worldwide. Lee et al (2012) quantify the ill-effects of inactivity and show that it caused $9 \%$ of premature mortality or more than 5.3 million of the 57 million deaths that occurred worldwide in $2008 .^{1}$

People benefit from even modest physical activity (PA): compared with inactive individuals, those who were active about 90 minutes per week lived three years longer (see Wen et al, 2011). Moreover, the practice of PA decreases not only the prevalence of non-communicable diseases (NCDs) but also long term care (LTC) dependency. ${ }^{2}$ In fact, physically active older adults are also more likely to perform better Instrumental Activities of Daily Living and Activities of Daily Living, to have a better functional health, lower risk of falling, and better cognitive function. PA delays entrance into dependency by six to seven years (see Shephard, 1991), and clearly has vast potential to improve health throughout the world. For its general health benefits and the potential decrease of health care costs, PA promotion is now a priority for governments and health agencies.

The goal of this paper is to analyze the effects of PA encouragement policies, such as uniform mass media campaigns, enhancing the social determinants of PA practice. To this end, we consider a community which is composed of two types of individuals who differ in their concern for PA. Additionally, we consider "social multiplier" effects in PA practice and, in particular, we assume that the marginal productivity of PA is increasing in the aggregated amount invested by the community. Social multiplier

\footnotetext{
${ }^{1}$ This figure equates to as many deaths as tobacco causes globally.

${ }^{2}$ See the survey of Blain et al. (2000), and the references therein.
} 
effects have been recently used to explain topics as diverse as criminal activity (Glaeser et al, 1996), welfare state participation (Bertrand et al, 2000), school achievement (Sacerdote, 2001), participation in retirement plans (Saez and Duflo, 2003), work and leisure (Alesina et al, 2005), and obesity (Trogdon et al, 2008). Note that an externality emerges since individuals do not account for the effect of their choices on the social multiplier effect, and hence on others' utility. Consequently, individual choices will not be optimal and there is therefore scope for government intervention.

Policies to promote PA are numerous and have been recently surveyed by Kohl et al (2012). Among theses policies, the informational approaches of community-wide and mass media campaigns, and short PA messages targeting key community sites are recommended (see the recent survey of Heath et al, 2012). In fact, while PA is globally accepted as health-enhancing for the full range of individuals' health statuses, less consensus exists regarding whether or not PA promoting policies should be publicized and implemented. For example, there is some discussion concerning the messages used to change PA behavior; in particular, whether those emphasizing the benefits of being active are more or less effective than those emphasizing the consequences of inactivity (see Latimer et al, 2010, or Gallagher and Updegraff, 2012).

We abstract from the implementation dimension of the problem and focus solely on PA recommendation policies, since we want to assess the relevance of the uniform PA recommendations presently used worldwide within mass media campaigns. Examples of such campaigns are the "Let's Move - Get Active" in the US, "Manger-Bouger" in France, "Find thirty every day" in Australia advising adults to do 30 minutes of daily moderate PA, and the "Change4life-150 minutes" of weekly PA in the UK. ${ }^{3}$ Importantly, the design of recommendations crucially depends on whether or not the government observes individuals' concerns for PA. When this is not observable, we suppose that

\footnotetext{
${ }^{3}$ For more on these campaigns check, respectively, http://www.letsmove.gov/get-active, www.mangerbouger.fr, www.findthirtyeveryday.com.au, and http://www.nhs.uk/Change4Life, all accessed on September 25, 2012. Similarly, there are uniform campaigns concerning nutrition, in general advising the intake of five portions of fruit and vegetables a day.
} 
the government implements a uniform policy. On the contrary, when this is observable, the government implements the First Best equilibrium.

The novelty of our approach is to take into account the peer effects existing in PA practice. Research suggests that the attitude of children and adolescents towards PA greatly depends on their parents' and peers' attitudes, as well as on their overall school environment (see among others, Eder and Parker, 1987, Wechsler et al, 2000, Smith, 2003, Voorhees et al, 2005, Robbins et al, 2008, Efrat, 2009, Babcock and Hartman, 2010, or Carrell et al, 2011). Additionally, according to a recent OECD (2010, p. 18) recommendation, "in the design and implementation of prevention policies special attention must be placed on the role of information, externalities and self-control issues, including the role of social multiplier effects (the clustering and spread within households and social networks)." What is more, researchers put forward whether or not it is the social contact inherent within PA programs for the elderly that creates a positive relationship between PA and cognition (see for example Renaud and Bherer, 2005). In their recent survey concerning PA in old age, Hirvensalo and Lintunen (2011, p. 18) conclude that, "the studies reviewed also highlighted the importance of social networks in maintaining participation." We incorporate this social dimension of PA into our model by assuming that individuals' benefit from PA is increasing in the aggregate average of PA in society.

In a setting with two types of individuals as regards their concern for PA, we contrast the Laissez Faire equilibrium with two government policies. In Section 2, we first assume that the government cannot observe types, defined as different preferences regarding PA, and enforces a uniform PA level (hereafter Government equilibrium). In Section 3, we then suppose that types are observable and therefore the government can implement the First Best. In this case, types may be interpreted as heterogenous PA benefits driven from different individual health conditions (or alternatively, age or gender). In fact, according to the medical literature, a lower level of PA is needed for the elderly, or for any individual with a low exercise capacity, if health risks are to be reduced. Also, while 30 to 60 minutes per day of moderate to vigorous intensity PA 
may be needed to decrease the risk of developing breast and colon cancers, 30 minutes of moderate intensity exercise five days per week reduces the risk of type-2 diabetes by $25 \%$ to $36 \%$ (see PAGAC, 2008). ${ }^{4}$ Still, government PA campaigns recommend uniform PA practice or, at most, provide different advice for children and adults.

We find that at the Laissez Faire PA individual choices are increasing in their concern for PA and in the social multiplier, as expected. The uniform government PA recommendation is above both types' individual choices if the social multiplier is high enough. Also, a uniform policy may improve the welfare of individuals the most concerned with PA, even when it reduces their level of PA. Indeed they benefit from the multiplicative effect of the increase in PA of individuals the least concerned with PA. Yet, the most unexpected result is that the welfare of the individuals the least concerned with PA may decrease in the social multiplier if they are not too numerous. This result is surprising because the direct effect of the social multiplier is a utility increase. However, such direct effect is dominated by the effect of imposing a too high level of PA, which results from a higher weight being given to the preference of individuals the most concerned with PA (a large number), to the detriment of those the least concerned with PA.

In contrast to the uniform policy, we find that the First Best PA recommendations are always above individuals' decisions, for all strengths of the social multiplier. Also, for individuals the most (resp: least) concerned with PA, the government recommendation is larger (resp: lower) than the uniform one. While the welfare of individuals the most concerned with PA increases in the social multiplier, the welfare of individuals the least concerned with PA may decrease in it. Therefore such a decrease is not due to the uniformity of the government intervention. Whatever their proportion, individuals

\footnotetext{
${ }^{4}$ See among others, Warburton et al (2006), regarding PA benefits in reducing the risk of several conditions, Barnett et al (2003) for reduction in falls and disability, Keysor (2003) for improved independence, McAuley et al (2005) for improved psychological well-being, and Colcombe and Kramer (2003), and the survey of Vogel et al (2009) for maintenance of cognitive vitality. Yet note that PA is recommended to all ages since the risk of NCDs starts in childhood (see Warburton et al, 2006, PAGAC, 2008, and WHO, 2010, among others).
} 
the most concerned with PA are better off at the Government equilibrium rather than at the First Best for a sufficiently high social multiplier.

Finally, for a sufficiently high social multiplier, both government interventions improve the welfare of the most concerned with PA but worsen the welfare of the least concerned individuals if they are not too many. More precisely, compared to the First Best, a uniform recommendation improves the welfare of those most concerned with PA more than it reduces the welfare of those least concerned.

The rest of the paper is organized as follows. In Section 2 we present our framework and contrast the Laissez Faire and Government equilibria. In Section 3 we contrast the First Best, where types are observable to the Laissez Faire and Government equilibria. A final section concludes. Proofs are gathered in the Appendices.

\section{The standard framework and uniform policy advice}

The economy is composed of individuals and the government. Individuals live for one period of time, of length normalized to 1, and they differ according to their concern $\alpha$ for PA. Indeed, we suppose the community to be composed of a fraction $p \in(0,1)$ of individuals with a concern $\alpha_{A}$ and $1-p$ with a concern $\alpha_{B}$.

Assumption 1. $1 / 2 \leq \alpha_{B}<\alpha_{A} \leq 1$.

Without loss of generality, we assume that type $A$ individuals have a greater concern for PA than type $B$ individuals. The assumption $1 / 2<\alpha_{B}$ can be relaxed but it guarantees that our results are not due to the use of exotic frameworks with huge heterogeneity. In particular, assuming a sufficiently large concern for PA ensures equilibria with positive levels of PA.

Individuals of type $i(i=A$ or $B)$ care about consumption $c_{i}$ and PA $\theta_{i}$. They care about PA because it is good for their health and because they enjoy it. ${ }^{5}$ The basic starting point of a social multiplier model is to assume that the marginal productivity

\footnotetext{
${ }^{5}$ These variables $\theta_{i}$ can alternatively be either a time or an amount of wealth devoted to PA.
} 
of PA is increasing in the amount chosen by one's peers. Then, as in Alesina et al (2005), we assume that utility is separable so that individuals maximize:

$$
\mathcal{U}_{i}=U\left(c^{i}\right)+\alpha_{i} H\left(\theta_{i}, \widehat{\theta}\right)
$$

where $\widehat{\theta}$ is the average amount of PA within the community. We assume $H_{1}\left(\theta_{i}, \widehat{\theta}\right)>0$, $H_{11}\left(\theta_{i}, \widehat{\theta}\right)<0$, and $H_{12}\left(\theta_{i}, \widehat{\theta}\right)>0$. In other words, PA increases health but at a decreasing rate, and the cross-partial reflects an increase in well-being driven from social interactions taking place during PA.

In contrast with standard models of network effects (Katz and Shapiro, 1985), PA $\theta$ can be viewed as a good which is consumed in variable quantities by heterogeneous individuals, and where the magnitude of the peer effects depends on the total quantity consumed across types, rather than on the total number of individuals in the economy. In addition, the value each individual receives on account of peer effects depends on the individual's consumption, as well as on the individual's type. In other words, we have type-dependent peer effects.

Following Alesina et al (2005) we use a specification of $\mathcal{U}_{i}$ where $U\left(c^{i}\right)=c^{i}$ is linear and $H\left(\theta_{i}, \widehat{\theta}\right)=2 \theta_{i}-\theta_{i}^{2}+\varepsilon \theta_{i} \widehat{\theta}$ is quadratic. ${ }^{6}$ As $c^{i}=1-\theta_{i}$, individuals' utility function is thus given by:

$$
\mathcal{U}_{i}=1-\theta_{i}+\alpha_{i}\left[2 \theta_{i}-\theta_{i}^{2}+\varepsilon \theta_{i} \widehat{\theta}\right]
$$

where the parameter $\varepsilon \in[0,1]$ measures the social multiplier. ${ }^{7}$

First we focus on the Laissez Faire (LF) equilibrium. Then, a type $i$ individual chooses the level of PA $\theta_{i}^{L F}$ that maximizes $\mathcal{U}_{i}$, as defined by (1). Individuals take as given the average amount of PA within the community $\widehat{\theta}$. Indeed, we assume that the individual's weight in the community is too small and thus they do not account for the effect of their individual choices on $\widehat{\theta}$. From the individual problems we obtain the

\footnotetext{
${ }^{6} H($.$) is the V($.$) of Alesina et al (2005, Section 5) when \nu_{0}=2, \nu_{1}=1$ and $\nu_{2}=\varepsilon$.

${ }^{7}$ Even though we focus on the social multiplier effects associated with PA practice the present setup is sufficiently general to be used in the analyzes of other goods and services where social multiplier effects occur, such as education or the use of new technologies.
} 
following FOCs:

$$
\theta_{A}^{L F}=1-\frac{1}{2 \alpha_{A}}+\frac{\varepsilon \widehat{\theta}}{2}
$$

and:

$$
\theta_{B}^{L F}=1-\frac{1}{2 \alpha_{B}}+\frac{\varepsilon \widehat{\theta}}{2}
$$

Irrespective of the individual's type, $\theta_{i}^{L F}$ is increasing in the average community

PA $\widehat{\theta}$. The (interior) equilibrium of the LF economy consists of a pair $\left(\theta_{A}^{L F}, \theta_{B}^{L F}\right) \in$ $(0,1) \times(0,1)$ which solves $(2 A),(2 B)$ and $\widehat{\theta}=p \theta_{A}^{L F}+(1-p) \theta_{B}^{L F}$. We can establish that:

\section{Proposition 1.}

The $L F$-equilibrium $\left(\theta_{A}^{L F}, \theta_{B}^{L F}\right) \in(0,1) \times(0,1)$ is given by:

$$
\theta_{A}^{L F}=\frac{2 \alpha_{B}\left(2 \alpha_{A}-1\right)-\varepsilon(1-p)\left(\alpha_{A}-\alpha_{B}\right)}{2(2-\varepsilon) \alpha_{A} \alpha_{B}}
$$

and:

$$
\theta_{B}^{L F}=\frac{2 \alpha_{A}\left(2 \alpha_{B}-1\right)+\varepsilon p\left(\alpha_{A}-\alpha_{B}\right)}{2(2-\varepsilon) \alpha_{A} \alpha_{B}}
$$

The optimal value $\theta_{A}^{L F}$ is larger than $\theta_{B}^{L F}$ and the two values are both increasing and convex functions of $\varepsilon$ with the same slopes.

Proof. See Appendix A.

Proposition 1 characterizes the LF-equilibrium in which those the most concerned with PA end up consuming more of it. Additionally, the higher the social multiplier the higher the equilibrium levels of PA.

We now focus on the Government $(\mathrm{G})$ equilibrium by considering a utilitarian government, which maximizes a weighted sum of individuals' utility $\mathcal{W}=p \mathcal{U}_{A}+(1-p) \mathcal{U}_{B}$, where $\mathcal{U}_{i}$ is given by $(1)$.

While the government knows the distribution of types in the community and recognizes that individuals have different preferences regarding PA, we suppose, in this section, that these are not observable and that it is too costly for the government to 
impose different levels of PA. Therefore, it chooses and enforces a uniform level $\theta^{G}$, irrespectively of individuals' concerns regarding PA.

We acknowledge that we abstract from the discussion about the government capability of enforcing PA recommendations and assume they are indeed enforced. In practice we tend to believe that policies relying on incentives may be more effective than enforcement policies which are much more costly to implement. Before returning to this discussion in the conclusion, we note that it is indeed possible for the government to impose PA. An example is the common practice of compulsory PA classes in schools, according to the age of the students. Compulsory PA could indeed also be a common practice in elderly care facilities, being enough to restrict licenses to the institutions fulfilling PA requirements. Yet for the time being note that PA enforcement for the overweight is being currently discussed in England, and Japan has enforced health treatments which include PA for those 40 years old and older who are over the maximum waist circumference authorized by the Metabo law. ${ }^{8}$

In contrast to individual behavior, the government internalizes the effect of individual PA levels on the community average level $\widehat{\theta}$ and, $\widehat{\theta}=\theta^{G}$. Thus, the government chooses the uniform level of PA $\theta^{G}$ that maximizes the following welfare function:

$$
\mathcal{W}=1-\theta+\Lambda\left[2 \theta-(1-\varepsilon) \theta^{2}\right]
$$

where $\Lambda=p \alpha_{A}+(1-p) \alpha_{B}$ and the FOC being:

$$
1-\widetilde{\varepsilon}_{G}=(1-\varepsilon) \theta^{G}
$$

where, according to Assumption 1, $\widetilde{\varepsilon}_{G}=1 /(2 \Lambda)<1$. We can state the following proposition:

\section{Proposition 2.}

The $G$-equilibrium $\theta^{G} \in(0,1]$ is given by:

\footnotetext{
${ }^{8}$ See for England http://www.telegraph.co.uk/health/healthnews/9777453/Obese-people-may-beforced-to-exercise-or-lose-benefits.html, and for Japan http://www.nytimes.com/2008/06/13/world/asi a/13fat.html both accessed on February 20, 2013.
} 


$$
\theta^{G}= \begin{cases}\frac{1-\widetilde{\varepsilon}_{G}}{1-\varepsilon} & \text { if } \quad \varepsilon<\widetilde{\varepsilon}_{G} \\ 1 & \text { if } \varepsilon \geq \widetilde{\varepsilon}_{G}\end{cases}
$$

The optimal value $\theta^{G}$ is an increasing and convex function of $\varepsilon$ for $\varepsilon<\widetilde{\varepsilon}_{G}$. This function is more convex than $\theta_{A}^{L F}$ and $\theta_{B}^{L F}$. Additionally, $\theta^{G}$ is larger than $\theta_{B}^{L F}$. Moreover, there exists a unique $\bar{\varepsilon}_{G} \in\left(0, \widetilde{\varepsilon}_{G}\right)$ such that $\theta^{G}$ is larger (resp: lower) than $\theta_{A}^{L F}$ if and only if $\varepsilon$ is larger (resp: lower) than $\bar{\varepsilon}_{G}$.

Proof. See Appendix B.

Results of Proposition 1 and 2 can be summarized by Figure 1, which plots the functions $\theta^{G}, \theta_{A}^{L F}$, and $\theta_{B}^{L F}$. These are increasing and convex in the social multiplier $\varepsilon$.

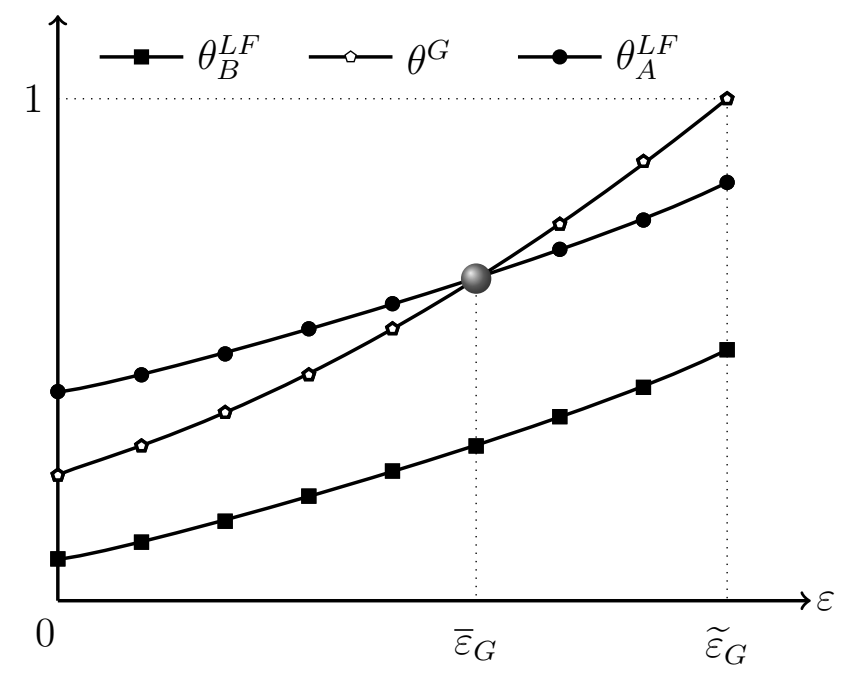

Figure 1: LF-equilibrium versus G-equilibrium

When the social multiplier is high enough $\left(\varepsilon>\bar{\varepsilon}_{G}\right)$ the PA level imposed by the government is above both individual types' equilibrium levels. Yet, when the social multiplier is not as strong, it is optimal for the government to impose a $\theta^{G}$ above type $B$ individuals' PA but below type $A$ individuals' PA.

Since neither type of individuals internalize the effects of the social multiplier, their choices have the same slope. On the contrary, the government internalizes the social multiplier effect, and therefore the stronger this effect the more the government is willing to increase PA practice. Consequently, $\theta^{G}$ is more convex than $\theta_{A}^{L F}$ and $\theta_{B}^{L F}$.

Finally, we focus on individuals' welfare. According to Appendix C, the welfare 
of type $i$ individuals at the LF-equilibrium, denoted $\mathcal{U}_{i}^{L F}$, and at the G-equilibrium, denoted $\mathcal{U}_{i}^{G}$, are:

$$
\begin{gathered}
\mathcal{U}_{i}^{L F}=1+\alpha_{i}\left(\theta_{i}^{L F}\right)^{2} \\
\mathcal{U}_{i}^{G}=1+\left[\left(1+\widetilde{\varepsilon}_{G}\right) \alpha_{i}-1\right] \theta^{G}
\end{gathered}
$$

Then, concerning type $A$ individuals welfare, we can establish that:

\section{Proposition 3.}

Both at the LF-equilibrium and at the $G$-equilibrium, $\mathcal{U}_{A}$ is an increasing and convex function of $\varepsilon$ and there exists a unique $\widehat{\varepsilon}_{A} \in\left(0, \bar{\varepsilon}_{G}\right)$ such that:

$$
\mid \begin{aligned}
& \mathcal{U}_{A}^{L F}>\mathcal{U}_{A}^{G} \quad \text { if } 0 \leq \varepsilon<\widehat{\varepsilon}_{A} \\
& \mathcal{U}_{A}^{L F}<\mathcal{U}_{A}^{G} \quad \text { if } \widehat{\varepsilon}_{A}<\varepsilon \leq \widetilde{\varepsilon}_{G}
\end{aligned}
$$

Proof. See Appendix C.

Proposition 3 states that whether type $A$ individuals are better off under the uniform government recommendation depends on the social multiplier. Its results are summarized in Figure 2.

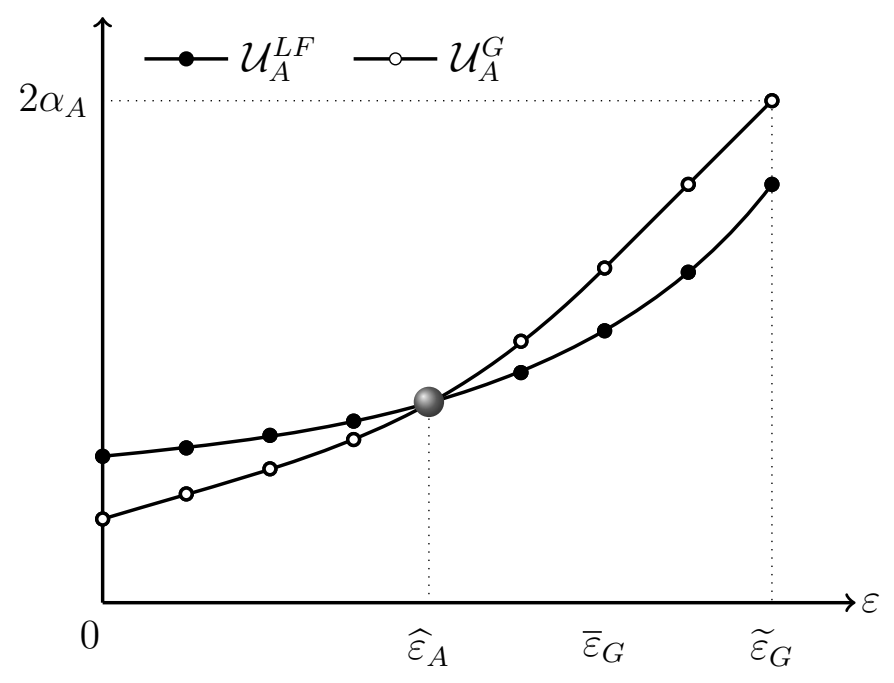

Figure 2: Welfare for type $A$ individuals

For low levels of the social multiplier $\left(\varepsilon<\widehat{\varepsilon}_{A}\right)$, type $A$ individuals are worse off under the G-equilibrium than under the LF-equilibrium. In this case, $\theta_{B}^{L F}<\theta^{G}<\theta_{A}^{L F}$ (Figure 1), but peer effects are not strong enough to multiply the effect of the increase 
of type $B$ individuals' PA and compensate for the reduction of type $A$ individuals' PA. On the contrary, if the social multiplier is high enough $\left(\varepsilon>\widehat{\varepsilon}_{A}\right)$, type $A$ individuals are better off under the government uniform policy $\theta^{G}$. Indeed, when $\varepsilon \in\left(\widehat{\varepsilon}_{A}, \bar{\varepsilon}_{G}\right)$, the reduction of type $A$ individuals' PA is more than compensated by the increase of type $B$ individuals' PA, whereas, when $\varepsilon>\bar{\varepsilon}_{G}$, peer effects are so beneficial that, additionally, it pays to increase type $A$ individuals' $\mathrm{PA}$ with respect to individual choices.

The function $\mathcal{U}_{A}^{G}$ is more convex than $\mathcal{U}_{A}^{L F}$ because the government takes into account the externality associated with the social multiplier, the importance of which is increasing in $\varepsilon$, while individuals do not.

Regarding the welfare of type $B$ individuals, and using the threshold $\widehat{p}=\min \left\{1, \alpha_{B}\left(2 \alpha_{B}-\right.\right.$ 1) $\left./\left[2\left(\alpha_{A}-\alpha_{B}\right)\left(1-\alpha_{B}\right)\right]\right\}$, we can establish that:

\section{Proposition 4.}

At the $L F$-equilibrium, $\mathcal{U}_{B}^{L F}$ is an increasing and convex function of $\varepsilon$.

At the $G$-equilibrium, $\mathcal{U}_{B}^{G}$ is an increasing and convex function of $\varepsilon$ for $p \leq \widehat{p}$ and a decreasing and concave function of $\varepsilon$ for $p>\widehat{p}$.

Moreover, there exists a unique $\bar{p} \leq \widehat{p}$ such that:

- if $p>\bar{p}$, then $\mathcal{U}_{B}^{L F}>\mathcal{U}_{B}^{G}$ for all $0 \leq \varepsilon \leq \widetilde{\varepsilon}_{G}$

- if $p \leq \bar{p}$, there exists a unique $\widehat{\varepsilon}_{B} \in\left(0, \widetilde{\varepsilon}_{G}\right)$ such that:

$$
\mid \begin{aligned}
& \mathcal{U}_{B}^{L F}>\mathcal{U}_{B}^{G} \quad \text { if } 0 \leq \varepsilon<\widehat{\varepsilon}_{B} \\
& \mathcal{U}_{B}^{L F} \leq \mathcal{U}_{B}^{G} \quad \text { if } \widehat{\varepsilon}_{B} \leq \varepsilon \leq \widetilde{\varepsilon}_{G}
\end{aligned}
$$

Proof. See Appendix D.

Proposition 4 states that the government does better for type $B$ individuals than the Laissez Faire if $(i)$ there are many type $B$ individuals to care about $(p \leq \bar{p})$, so that the government weights enough their welfare and chooses a level of PA in accordance with their weaker preference for it; and (ii) if peer effects are strong enough $\left(\widehat{\varepsilon}_{B} \leq \varepsilon \leq \widetilde{\varepsilon}_{G}\right)$. 
On the contrary, the government always does better for type $A$ individuals as long as peer effects are strong enough (Proposition 3).

In order to understand the results of Propositions 3 and 4, it is necessary to understand why $\mathcal{U}_{B}^{G}$ increases in $\varepsilon$ for sufficiently low values of $p$ and decreases for sufficiently large values of $p$, whereas $\mathcal{U}_{A}^{G}$ increases in $\varepsilon$ whatever $p$. Several direct and indirect effects are at play. First it helps to note that $\mathcal{U}_{i}^{G}$ is a function of $\theta_{\varepsilon, p}^{G}$, given $\varepsilon$ and $p$. In fact, at $\hat{\theta}=\theta_{\varepsilon, p}^{G},(1)$ is simplified to:

$$
\mathcal{U}_{i}^{G}\left(\varepsilon, \theta_{\varepsilon, p}^{G}\right)=1+\left[2 \alpha_{i}-1\right] \theta_{\varepsilon, p}^{G}-\alpha_{i}(1-\varepsilon)\left[\theta_{\varepsilon, p}^{G}\right]^{2}
$$

It is clear that the function $\mathcal{U}_{i}^{G}($.$) is concave in \theta^{G}$, increasing towards the maximum and afterwards decreasing, for both types $(i=A, B)$. Its maximum value is attained at:

$$
\theta_{i}^{\star}=\frac{2 \alpha_{i}-1}{2 \alpha_{i}(1-\varepsilon)}
$$

This maximum corresponds to the G-equilibrium of Proposition 2 with $p=1$ and $p=0$ for respectively type- $A$ and type- $B$ individuals, i.e., $\theta_{A}^{\star}=\theta_{\varepsilon, 1}^{G}$ and $\theta_{B}^{\star}=\theta_{\varepsilon, 0}^{G}$.

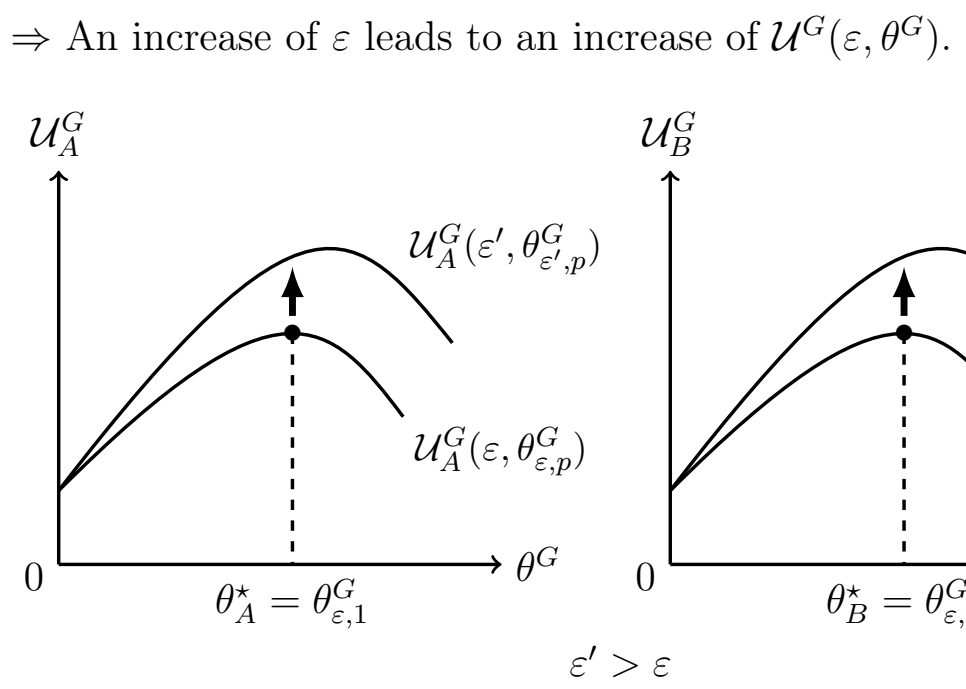$$
\mathcal{U}_{B}^{G}
$$

$\varepsilon^{\prime}>\varepsilon$

Figure 3: Direct effect

A first direct effect is that $\mathcal{U}_{i}^{G}($.$) is increasing in \varepsilon>0$, as it follows from (5). Graphically, if $\mathcal{U}_{i}^{G}($.$) is plotted against \theta^{G}$, an increase in $\varepsilon$ shifts the curve $\mathcal{U}_{i}^{G}($. upwards, as represented in Figure 3. 
Other indirect effects are translated into a movement along the curve $\mathcal{U}_{i}^{G}($.$) as \varepsilon$, and $p$ vary. Indeed, $\theta_{\varepsilon, p}^{G}$ increases in $\varepsilon$, and $p$ as:

$$
\theta_{\varepsilon, p}^{G}=\frac{2 \alpha_{B}-1+2 p\left(\alpha_{A}-\alpha_{B}\right)}{2(1-\varepsilon)\left[\alpha_{B}+p\left(\alpha_{A}-\alpha_{B}\right)\right]}
$$

Such indirect effects are represented in Figure 4.

$\Rightarrow$ An increase of $\varepsilon$ leads to an increase of $\theta_{\varepsilon, p}^{G}$.

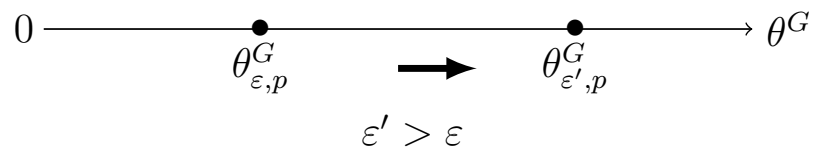

$\Rightarrow$ An increase of $p$ leads to an increase of $\theta_{\varepsilon, p}^{G}$.

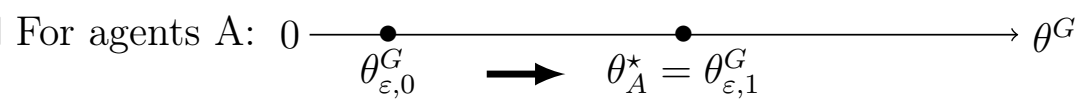

For agents B: $0 \longrightarrow \stackrel{\bullet}{\theta_{B}^{\star}}=\theta_{\varepsilon, 0}^{G} \longrightarrow \stackrel{\bullet}{\rightarrow} \theta_{\varepsilon, 1}^{G} \theta^{G}$

Figure 4: Indirect effects

If $\theta_{\varepsilon, p}^{G}<\theta_{i}^{\star}$, an increase in $\varepsilon$ leads to a movement along $\mathcal{U}_{i}^{G}($.$) in its increasing interval$ and therefore such an indirect effect reinforces the first direct effect. Undoubtedly, $\mathcal{U}_{i}^{G}($.$) increases. This is the case for type A$ individuals, given that $\theta_{A}^{\star}$ takes its maximum at $p=1$. On the contrary, if $\theta_{\varepsilon, p}^{G}>\theta_{i}^{\star}$, an increase in $\varepsilon$ leads to a movement along $\mathcal{U}_{i}^{G}($.$) in its decreasing interval, which undermines the direct effect of increasing \mathcal{U}_{i}^{G}($.$) .$ This is the case for type $B$ individuals, given that $\theta_{B}^{\star}$ takes its maximum at $p=0$. Figures 5 and 6 summarize the forces at work.

In Figure $5, \mathcal{U}_{A}^{G}$ is represented as a function of the government policy $\theta^{G}$. As already shown, this function is concave and its maximum value is attained at $\theta_{\varepsilon, 1}^{G}$, the level of PA the government would had chosen if there were no type $B$ individuals. Since $\theta^{G}$ is increasing in $p$, for interior values of $p$ the government optimal choice must be to the left of $\theta_{\varepsilon, 1}^{G}$. Now let us observe the two effects when the social multiplier increases from $\varepsilon$ to $\varepsilon^{\prime}$. The direct effect shifts $\mathcal{U}_{A}^{G}$ up in conformity with $\mathcal{U}_{A}^{G}\left(\varepsilon^{\prime}, \theta_{\varepsilon^{\prime}, p}^{G}\right)>\mathcal{U}_{A}^{G}\left(\varepsilon, \theta_{\varepsilon, p}^{G}\right)$. The indirect effect also increases $\mathcal{U}_{A}^{G}$ since the government choice $\theta^{G}$ increases in the 
increasing branch of $\mathcal{U}_{A}^{G}$ (i.e., $\theta_{\varepsilon, p}^{G}<\theta_{\varepsilon^{\prime}, p}^{G}<\theta_{A}^{\star}$ ). Therefore, both effects lead to a increases of type $A$ individuals' welfare.

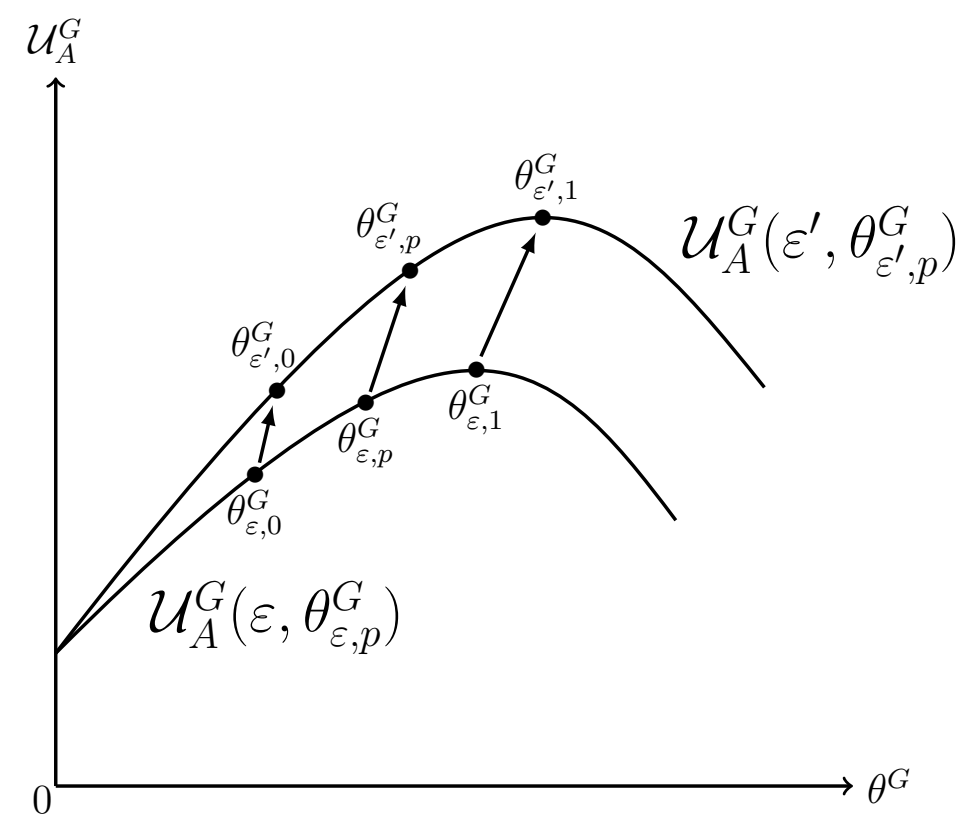

Figure 5: Unambiguous impact of $\varepsilon$ on type $A$ individuals' welfare

Now, we consider Figure 6 which plots the analogous function for type $B$ individuals.

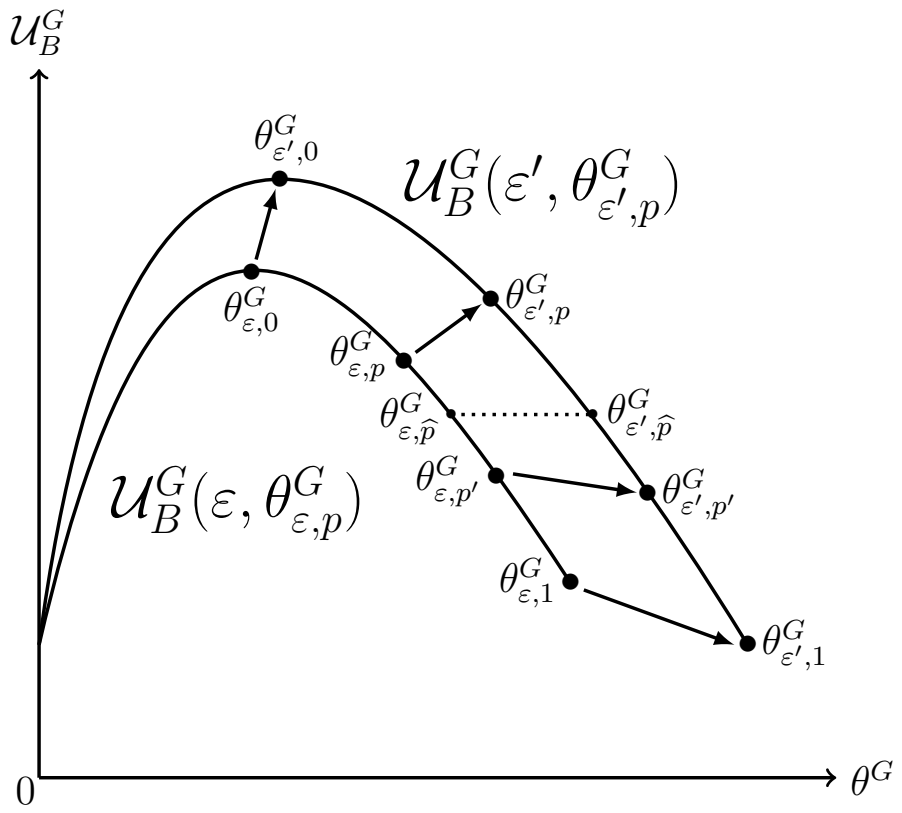

Figure 6: Ambiguous impact of $\varepsilon$ on type $B$ individuals' welfare

The maximum value of $\mathcal{U}_{B}^{G}$ is attained at $\theta_{\varepsilon, 0}^{G}$. Since $\theta^{G}$ is increasing in $p$, for interior values of $p$ the government optimal choice must be to the right of $\theta_{\varepsilon, 0}^{G}$. Now let us observe the two effects when the social multiplier increases from $\varepsilon$ to $\varepsilon^{\prime}$. The 
direct effect shifts $\mathcal{U}_{B}^{G}$ up in conformity with $\mathcal{U}_{B}^{G}\left(\varepsilon^{\prime}, \theta_{\varepsilon^{\prime}, p}^{G}\right)>\mathcal{U}_{B}^{G}\left(\varepsilon, \theta_{\varepsilon, p}^{G}\right)$. Importantly, the indirect effect on $\mathcal{U}_{B}^{G}$ is now ambiguous since the government choice $\theta^{G}$ increases in the decreasing branch of $\mathcal{U}_{B}^{G}$ (i.e., $\theta_{B}^{\star}<\theta_{\varepsilon, p}^{G}<\theta_{\varepsilon^{\prime}, p}^{G}$ ). The intensity of such an effect depends on $p$. If $p$ is low enough $(p<\widehat{p})$, then $\theta_{\varepsilon, p}^{G}$ is sufficiently near $\theta_{B}^{\star}$ implying that the indirect effect is weaker than the direct one. Consequently, type $B$ individuals' welfare ends up increasing. On the contrary, if $p$ is sufficiently high $\left(p^{\prime}>\widehat{p}\right)$, then the decreasing slope of $\mathcal{U}_{B}^{G}($.$) in \theta_{\varepsilon, p^{\prime}}^{G}$ is too important and thus the direct effect is completely neutralized by the indirect one. Consequently, an increase of $\varepsilon$ leads to a decrease of type $B$ individuals' welfare.

Results of Proposition 4 are illustrated in Figure 7.
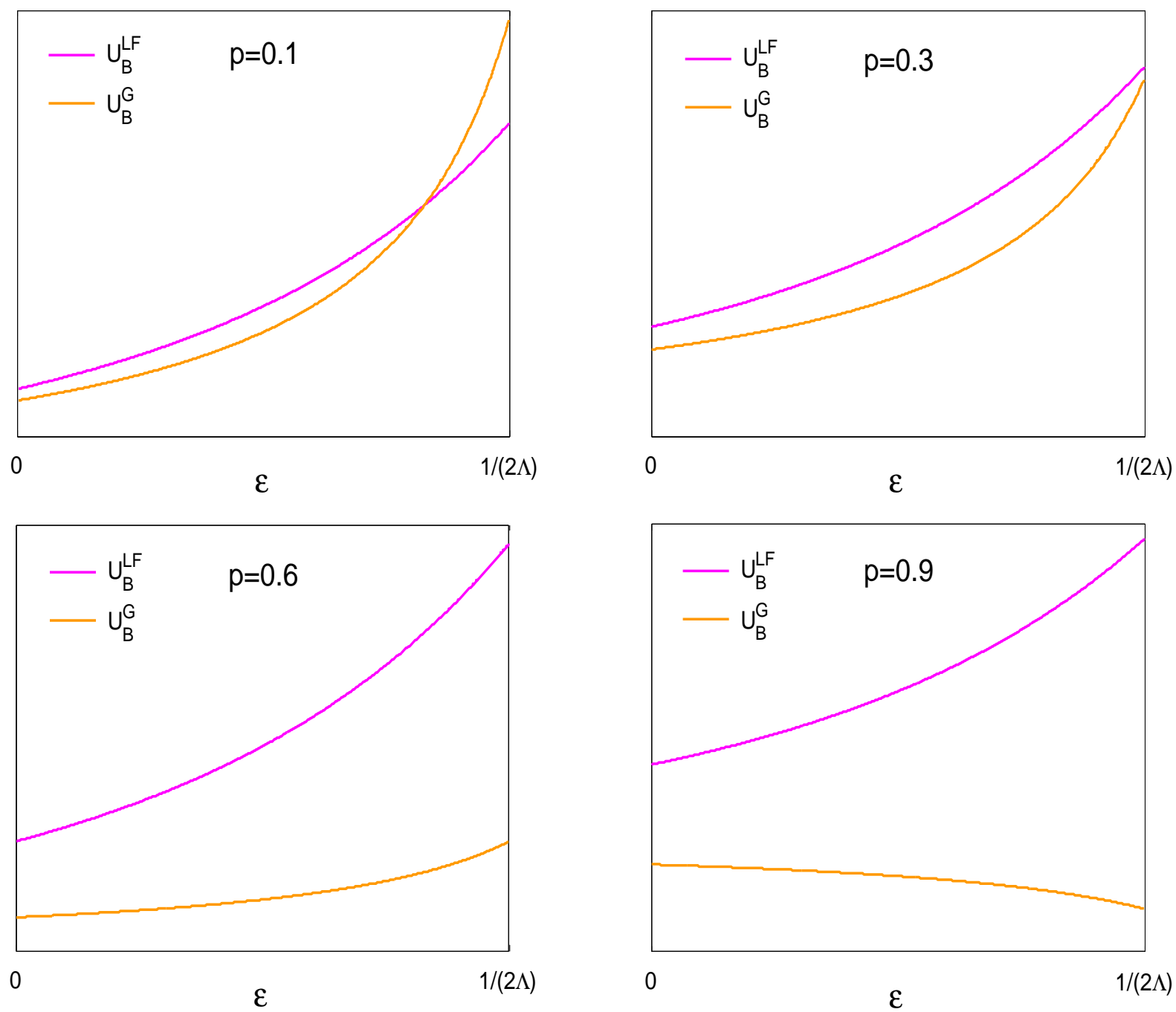

Figure 7: Type $B$ individuals' welfare according to $p_{A}$

This figure plots type $B$ individuals' welfare under the LF-equilibrium (purple line) 
and under the $\mathrm{G}$-equilibrium (orange line) as a function of the social multiplier. In this example we assume $\alpha_{A}=0.8$ and $\alpha_{B}=0.6$. The difference among the four figures relies on the value assumed for $p$, the proportion of individuals with the highest concern for PA. We have assumed, from the top-left to the bottom-right, $p=0.1, p=0.3, p=0.6$, and $p=0.9$.

Two remarks can be made. First, in the four examples, $\mathcal{U}_{B}^{L F}$ is increasing in the social multiplier whereas $\mathcal{U}_{B}^{G}$ is decreasing for $p=0.9$. This is precisely the result we discuss above, i.e., when type $A$ individuals are too many the government gives little weight to type $B$ individuals' preferences and imposes such a high level of PA that type $B$ individuals can end up worse off. Second, for sufficiently low levels of $p$, individuals are better off under the LF-equilibrium than under the government policy, unless peer effects become important. An illustration is provided for $p=0.1$.

According to Appendix E, the threshold $\widehat{\varepsilon}_{B}$ increases in $p$ and $\left.\widehat{\varepsilon}_{B}\right|_{p=0}=0$ while $\widehat{\varepsilon}_{A}$ decreases in $p$ and $\left.\widehat{\varepsilon}_{A}\right|_{p=1}=0$. Then, there exists a unique threshold $\underline{p} \in(0, \bar{p})$ such that $\widehat{\varepsilon}_{A}$ is larger (resp: lower) than $\widehat{\varepsilon}_{B}$ if and only if $p$ is lower (resp: larger) than $p$. Using $\underline{p}$ and Propositions 3 and 4 , we can state Corollary 1.

\section{Corollary 1.}

The G-equilibrium constitutes a Pareto improvement if and only if $(p<\underline{p}$ and $\left.\varepsilon>\widehat{\varepsilon}_{A}\right)$ or $\left(\underline{p}<p<\bar{p}\right.$ and $\left.\varepsilon>\widehat{\varepsilon}_{B}\right)$.

Both individual types are worse off under the G-equilibrium if and only if $(p<\underline{p}$ and $\left.\varepsilon<\widehat{\varepsilon}_{B}\right)$ or $\left(\underline{p}<p\right.$ and $\left.\varepsilon<\widehat{\varepsilon}_{A}\right)$.

\section{Proof. See Appendix E.}

Corollary 1 states the conditions under which the government should undoubtedly intervene and the conditions under which there is no role for government intervention because both types end up worse off. When the proportion of type $A$ individuals is not too high and peer effects are strong enough, both types of individuals are better off under the government policy. In this case, for sufficiently few type $A$ individuals, i.e., $p<\underline{p}$, type $B$ individuals are better off under the government policy at lower peer 
effects than are type $A$ individuals (because $\widehat{\varepsilon}_{B}<\widehat{\varepsilon}_{A}$ ). If, instead, type $A$ individuals are sufficiently numerous, i.e., $\underline{p}<p<\bar{p}$, it is those individuals who are better off under the government policy at lower peer effects (because $\widehat{\varepsilon}_{A}<\widehat{\varepsilon}_{B}$ ). If, on the other hand, peer effects are too weak then both individuals are worse off under the G-equilibrium and therefore there is no role for government intervention.

We have shown that the existence of a social multiplier is not a reason per se to justify government intervention. This result is due to the uniform PA level imposed on both individual types, which is a common practice of government PA (mass media) campaigns such as the ones discussed in the introduction. Our contribution aims to highlight that if the social multiplier associated with PA is low, then the government may indeed harm all in society by advising a uniform level of PA.

\section{The case with public information}

In this section we suppose that the government can implement the First Best. Thus, we assume that individual types are observable and that it is possible to enforce different PA levels. Such an exercise is only relevant as a benchmark since in the real world individuals' types are not observable. Nevertheless, there are situations in which the government can observe individual types. Returning to the PA campaigns discussed in the introduction, it is presently common practice to advise children to perform double the recommended PA for adults. We can interpret observable types as children and adults, and we abstract from any heterogeneity within the same type.

The First Best (FB) equilibrium consists of the government choices of $\theta_{A}^{F B}$ and $\theta_{B}^{F B}$ that maximize $\mathcal{W}=p \mathcal{U}_{A}+(1-p) \mathcal{U}_{B}$, with $\widehat{\theta}=p \theta_{A}^{F B}+(1-p) \theta_{B}^{F B}$.

Let $\delta=4 \alpha_{A} \alpha_{B}-(1-p)\left(\alpha_{A}-\alpha_{B}\right)\left(1+2 \alpha_{B}\right)$ and using the two following thresholds:

$$
\bar{\varepsilon}_{A}=\frac{-\delta+\sqrt{\delta^{2}+8 p(1-p)\left(\alpha_{A}-\alpha_{B}\right)^{2} \alpha_{B}}}{2 p(1-p)\left(\alpha_{A}-\alpha_{B}\right)^{2}} \quad \text { and } \quad \bar{\varepsilon}_{B}=\frac{1}{\alpha_{B}+\Lambda}
$$

we establish that: 


\section{Proposition 5.}

The $F B$-equilibrium $\left(\theta_{A}^{F B}, \theta_{B}^{F B}\right) \in(0,1] \times(0,1]$ is given by:

$$
\theta_{A}^{F B}= \begin{cases}\widetilde{\theta}_{A}^{F B}=\frac{2 \alpha_{B}\left(2 \alpha_{A}-1\right)-\varepsilon(1-p)\left(\alpha_{A}-\alpha_{B}\right)\left(1+2 \alpha_{B}\right)}{4 \alpha_{A} \alpha_{B}-4 \alpha_{A} \alpha_{B} \varepsilon-p(1-p)\left(\alpha_{A}-\alpha_{B}\right)^{2} \varepsilon^{2}} & \text { if } \varepsilon<\bar{\varepsilon}_{A} \\ 1 & \text { if } \varepsilon \geq \bar{\varepsilon}_{A}\end{cases}
$$

and:

$$
\theta_{B}^{F B}= \begin{cases}\widetilde{\theta}_{B}^{F B}=\frac{2 \alpha_{A}\left(2 \alpha_{B}-1\right)+\varepsilon p\left(\alpha_{A}-\alpha_{B}\right)\left(1+2 \alpha_{A}\right)}{4 \alpha_{A} \alpha_{B}-4 \alpha_{A} \alpha_{B} \varepsilon-p(1-p)\left(\alpha_{A}-\alpha_{B}\right)^{2} \varepsilon^{2}} & \text { if } \varepsilon<\bar{\varepsilon}_{A} \\ \bar{\theta}_{B}^{F B}=\frac{2 \alpha_{B}-1+\varepsilon p\left(\alpha_{A}+\alpha_{B}\right)}{2 \alpha_{B}[1-\varepsilon(1-p)]} & \text { if } \bar{\varepsilon}_{A} \leq \varepsilon<\bar{\varepsilon}_{B} \\ 1 & \text { if } \varepsilon \geq \bar{\varepsilon}_{B}\end{cases}
$$

The optimal values $\widetilde{\theta}_{A}^{F B}, \widetilde{\theta}_{B}^{F B}$ and $\bar{\theta}_{B}^{F B}$ are both increasing and convex functions of $\varepsilon$. Moreover, $\theta_{A}^{F B}$ is larger than $\theta_{B}^{F B}, \theta_{A}^{F B}$ is larger than $\theta_{A}^{L F}, \theta_{B}^{F B}$ is larger than $\theta_{B}^{L F}, \theta^{G}$ is lower than $\theta_{A}^{F B}$, and $\theta^{G}$ is larger than $\theta_{B}^{F B}$.

Proof. See Appendix F.

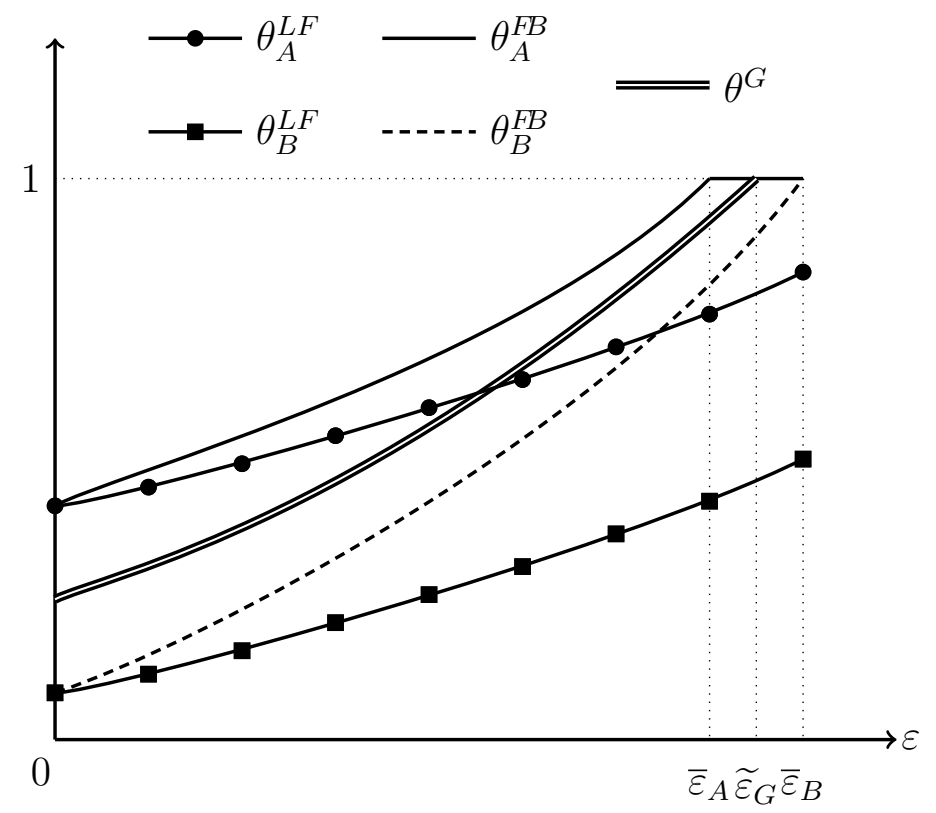

Figure 8: FB-equilibrium versus LF-equilibrium and G-equilibrium

Figure 8 plots $\theta_{A}^{F B}, \theta_{B}^{F B}, \theta_{A}^{L F}, \theta_{B}^{L F}$, and $\theta^{G}$. For each type, the FB-equilibrium PA level is above the respective LF-equilibrium value. The positive externality as- 
sociated with the social multiplier implies that individuals do too little PA in the LF-equilibrium. Also, $\theta_{A}^{F B}$ and $\theta_{B}^{F B}$ are increasing in the social multiplier. Moreover, as discrimination is feasible, the government chooses to increase (resp: decrease) type $A$ (resp: type $B$ ) individuals' $\mathrm{PA}$ relative to $\theta^{G}$, i.e., $\theta_{A}^{F B}>\theta^{G}>\theta_{B}^{F B}$. In a sense, the government is better able to take into consideration individual preferences.

Using the fact that $\bar{\varepsilon}_{A}<\widetilde{\varepsilon}_{G}<\bar{\varepsilon}_{B}$ and substituting in (1) the values $\theta_{A}^{F B}$ and $\theta_{B}^{F B}$ as defined above allow us to obtain:

$\mathcal{U}_{A}^{F B}=\left\{\begin{array}{l}\widetilde{\mathcal{U}}_{A}^{F B}=1+\left(\frac{\alpha_{B}\left(2 \alpha_{A}-1\right)}{\alpha_{A}+\alpha_{B}}\right) \widetilde{\theta}_{A}^{F B}+\left(\frac{\alpha_{A}\left(\alpha_{A}-\alpha_{B}\right)(1-\varepsilon p)}{\alpha_{A}+\alpha_{B}}\right)\left(\widetilde{\theta}_{A}^{F B}\right)^{2} \text { if } \varepsilon<\bar{\varepsilon}_{A} \\ \overline{\mathcal{U}}_{A}^{F B}=\alpha_{A}\left[1+\varepsilon p+(1-p) \varepsilon \bar{\theta}_{B}^{F B}\right] \text { if } \bar{\varepsilon}_{A} \leq \varepsilon \leq \widetilde{\varepsilon}_{G}\end{array}\right.$

and:

$\mathcal{U}_{B}^{F B}=\left\{\begin{array}{l}\widetilde{\mathcal{U}}_{B}^{F B}=1+\left(\frac{\alpha_{A}\left(2 \alpha_{B}-1\right)}{\alpha_{A}+\alpha_{B}}\right) \widetilde{\theta}_{B}^{F B}-\left(\frac{\alpha_{B}\left(\alpha_{A}-\alpha_{B}\right)[1-\varepsilon(1-p)]}{\alpha_{A}+\alpha_{B}}\right)\left(\widetilde{\theta}_{B}^{F B}\right)^{2} \text { if } \varepsilon<\bar{\varepsilon}_{A} \\ \overline{\mathcal{U}}_{B}^{F B}=1+\left(\frac{2 \alpha_{B}-1-\varepsilon p\left(\alpha_{A}-\alpha_{B}\right)}{2}\right) \bar{\theta}_{B} \text { if } \bar{\varepsilon}_{A} \leq \varepsilon \leq \widetilde{\varepsilon}_{G}\end{array}\right.$

We can thus establish:

\section{Proposition 6.}

The optimal values $\widetilde{\mathcal{U}}_{A}^{F B}$ and $\overline{\mathcal{U}}_{A}^{F B}$ are increasing and convex functions of $\varepsilon$. The optimal value $\widetilde{\mathcal{U}}_{B}^{F B}$ is an increasing function for sufficiently low values of $\varepsilon$, whereas $\overline{\mathcal{U}}_{B}^{F B}$ is an increasing function for sufficiently low values of $p$ but a decreasing one for sufficiently high values of $p$.

Moreover, when $\varepsilon$ is sufficiently low, then $\mathcal{U}_{A}^{F B}$ is larger than $\mathcal{U}_{A}^{G}$ whereas $\mathcal{U}_{A}^{F B}$ is lower than $\mathcal{U}_{A}^{G}$ for sufficiently large values of $\varepsilon$. Also, when $\varepsilon$ is sufficiently low or sufficiently large, then $\mathcal{U}_{B}^{F B}$ is larger than $\mathcal{U}_{B}^{G}$.

Proof. See Appendix G.

We comment on Proposition 6 above with the help of a numerical illustration where we suppose $\alpha_{A}=0.9, \alpha_{B}=0.6$, and $p=0.7$. 


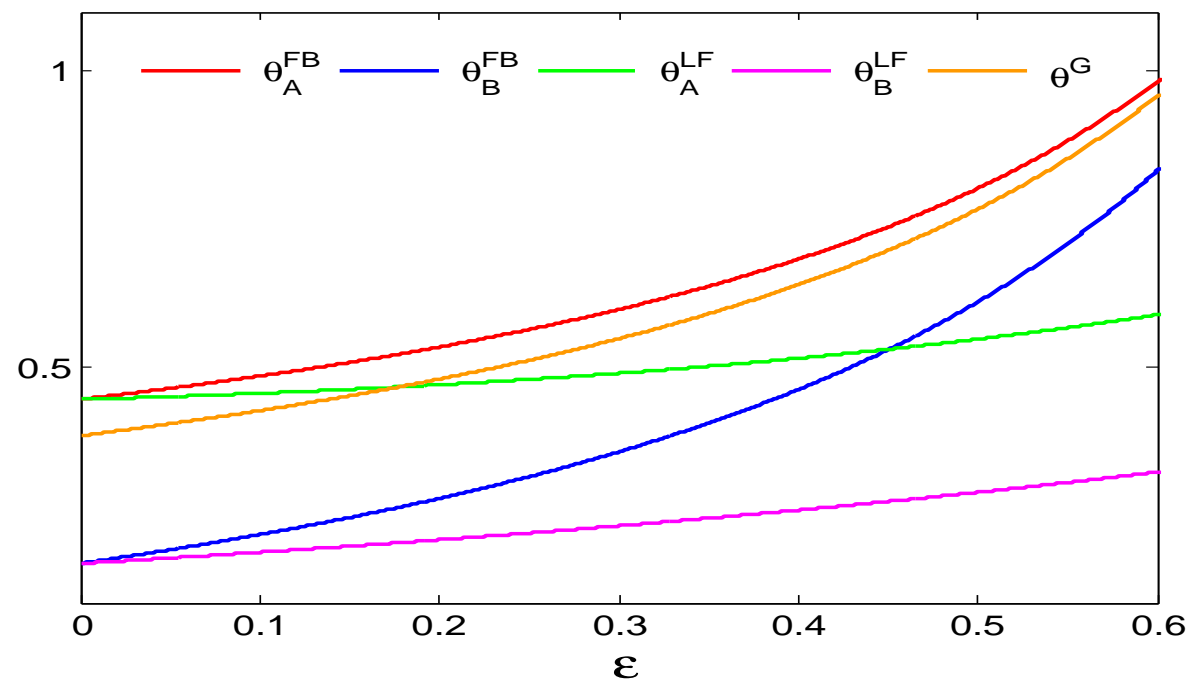

Figure 9: FB-equilibrium versus LF-equilibrium and G-equilibrium

In Figure 9 we plot the PA choices $\theta_{A}^{L F}, \theta_{B}^{L F}, \theta_{A}^{F B}, \theta_{B}^{F B}$, and $\theta^{G}$. It can be verified that we recover the proprieties established in Proposition 5 and Figure 8.

Figures 10 and 11 represent, respectively, type $A$ and $B$ individuals' welfare under the three scenarios LF-equilibrium, G-equilibrium, and FB-equilibrium. In these figures, the graphs on the left represent the welfare up to the threshold $\bar{\varepsilon}_{A}$. At the beginning the difference between the curves is not clear, and we therefore present on the right the same curves zoomed for the lowest values of $\varepsilon$.
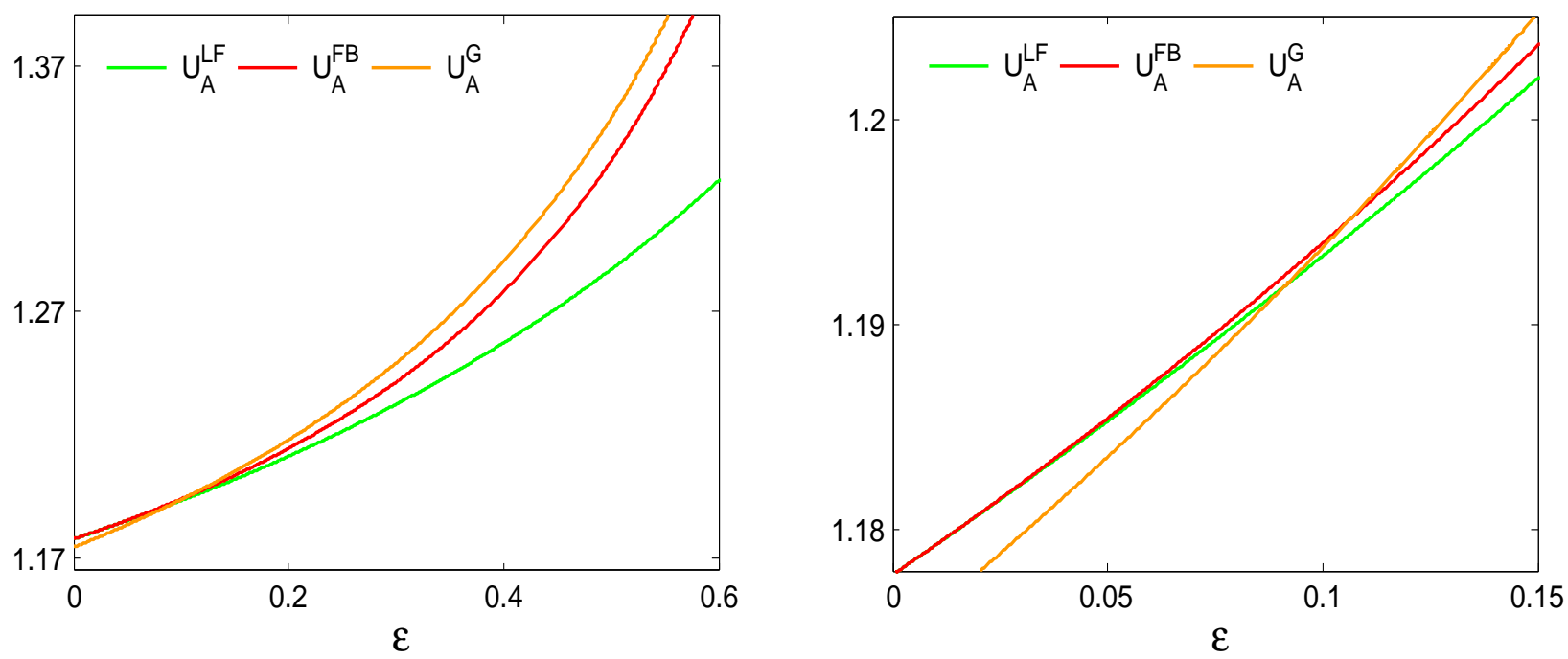

Figure 10: Type $A$ individuals' welfare

In fact, the welfare of First Best is always larger (resp: lower) than the welfare of Laissez Faire for individuals of type $A$ (resp: type $B$ ). We can also highlight that, first, for very low levels of the social multiplier, both types are worse off under the G- 

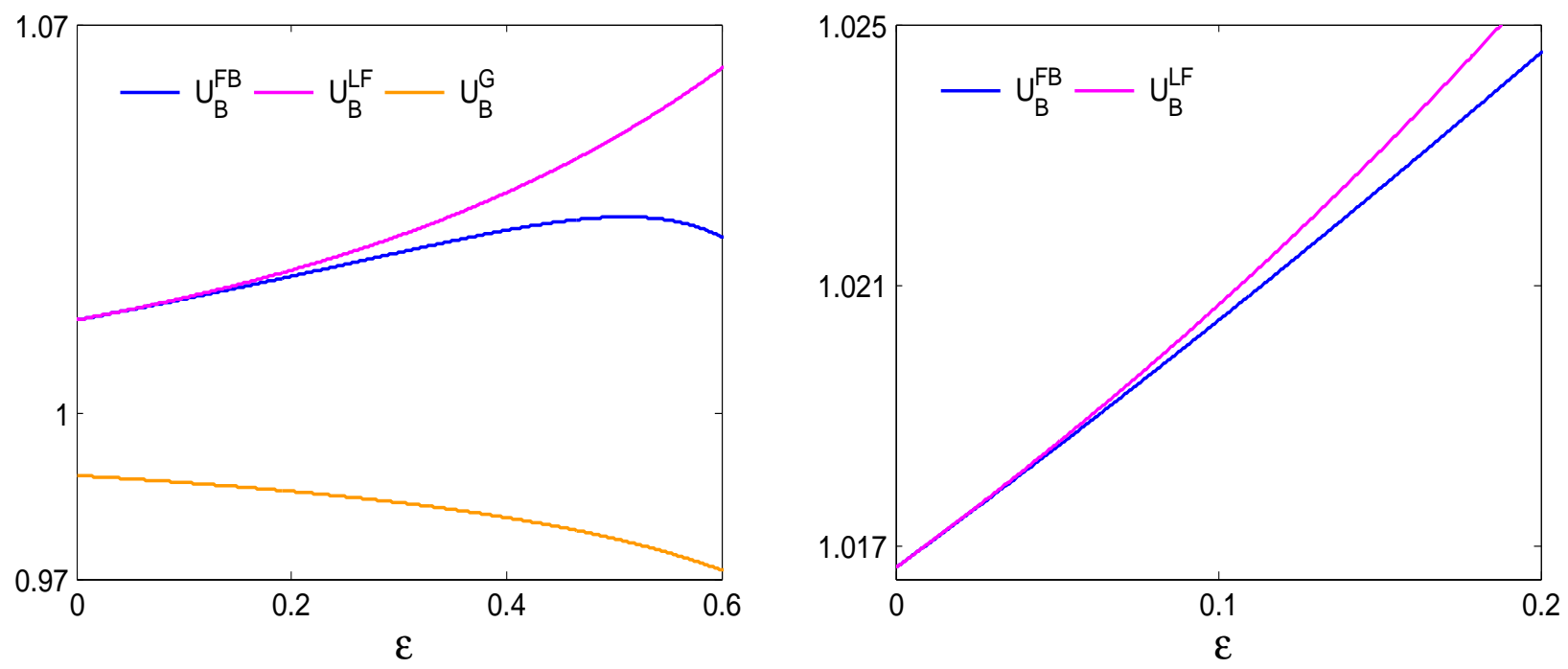

Figure 11: Type $B$ individuals' welfare

equilibrium than under the LF-equilibrium, in accordance with Corollary 1. Second, type $A$ individuals benefit from any government intervention and mainly from the G-equilibrium approach for high levels of the social multiplier, in accordance with Proposition 6. In fact, they benefit from the general increase in PA imposed on society without compromising a decrease in the consumption of other goods. This effect is stronger under the $\mathrm{G}$-equilibrium where a higher level of $\mathrm{PA}$ is imposed to type $B$ individuals. Third, for type $A$ individuals, the benefit associated with the government policies is most important for high levels of the social multiplier. Finally, the increase in type $A$ individuals' welfare is made at the cost of imposing a high level of PA to type $B$ individuals. Indeed type $B$ individuals are worse off under any of the two government policies than under the LF-equilibrium. Interestingly, their welfare - not only at the G-equilibrium but also at the FB-equilibrium - is decreasing even for higher levels of the social multiplier. In fact, a high social multiplier makes the government more willing to impose a higher level of PA because the positive externality effects become more important. Yet, this is done at the cost of a decrease of type $B$ individuals' welfare. 


\section{Conclusion}

We analyze the effects of PA encouragement policies when individuals differ with respect to their concern for PA and in the presence of a social multiplier effect in PA practice. If the social multiplier is strong enough, we find that uniform and First Best policies increase the welfare of the individuals the most concerned with PA, at the cost of a decrease in the welfare of those the least concerned when they are not too many. But, compared to the First Best, a uniform recommendation improves the welfare of those most concerned with PA more than it reduces the welfare of those least concerned. Moreover, the welfare of those the least concerned with PA may even be decreasing in the strength of the social multiplier, both under a uniform policy and under the First Best policy. Indeed, the social multiplier may be so strong that it may be welfare improving to increase the PA level to a degree that it harms the least concerned individuals but greatly benefits the most concerned individuals.

This paper can be extended in several directions. First, we focus on an aggregate externality, i.e., the social multiplier effect, and therefore individuals take as given the aggregate average of PA. Alternatively, we could assume that individuals perceive the impact of their choices on others' decisions with obvious consequences for public intervention. We believe it is worth developing a network setup in which individuals have social interactions with some individuals but not with others to encompass the latter possibility. Second, we analyze only one of many possible government interventions aiming at an increase in PA. Many others include provision of sporting infrastructures, revision of urban planning to ensure that walking, cycling, and other forms of active lifestyles are accessible and safe, or to encourage PA networks, among others. Additionally, PA could be seen as voluntary contributions towards a public good, say general health in the population or PA infrastructures. Finally, along our analyses we have considered that PA levels are enforced and verifiable but have neglected the discussion about its implementation. While the First Best policy could be decentralized by means of taxes/subsidies on PA, this is not necessarily the case for the uniform policy. A com- 
plement to our analyses could consider an incentive compatible problem assuming the concern for PA is not observable. In such a case, the government could implement a second best approach by offering a menu of contracts among which individuals would self-select. Alternatively, the government could make use of other individual characteristics correlated with PA concern as, for example, age. In this regard "tagging" could be used (see for example, Cremer et al., 2010, for a recent contribution, and for a survey). Finally, a potentially relevant alternative assumption is to consider that even the individuals themselves are not fully aware of the benefits of PA for their health. Bringing uncertainty to the present setup opens the door to explore the benefits of information disclosure mechanisms for health (and welfare) improvement (see, among others, Bardey and De Donder, 2012, for the use of genetic testing and its implications on prevention behaviors).

More specifically, recent studies have explored barriers to PA in LTC settings (see the survey of Benjamin et al, 2013), which can occur at the individual, organizational, and environmental levels. Reported barriers include seniors' poor health, fear of falling, and a past history of sedentary lifestyles; organizational challenges such as inadequate staffing levels and institutional care routines; and environmental realities such as lack of designated spaces and equipment for PA. Future studies targeting PA interventions for residents living in LTC are needed to address these multiple levels of influence. Importantly, our study shows how uniform policies may differently affect heterogenous populations, and how important it is to carefully implement adequate levels of PA for each target population. In particular, we find our results useful in considering PA policies targeting the elderly, which have, in our view, important consequences for the provision of LTC.

\section{References}

[1] Alesina, A., Glaeser, E. and B. Sacerdote, 2005, "Work and Leisure in the U.S. and Europe: why so different?", NBER Working Papers No. 11278. 
[2] Babcock, P. and J. Hartman, 2010, "Networks and workouts: treatment status specific peer effects in a randomized field experiment", NBER Working Paper No. 16581.

[3] Bardey, D. and P. De Donder, 2012, "Genetic testing with primary prevention and moral hazard", CEPR Discussion Papers No. 8977.

[4] Barnett, A., Smith, B., Lord, S.R., Williams, M. and A. Baumand, 2003, "Community-based group exercise improves balance and reduces falls in at-risk older people: a randomized controlled trial", Age and Ageing, 32, 407-414.

[5] Benjamin, K., Edwards, N., Ploeg, J. and F. Legault, 2013, "Barriers to physical activity and restorative care for residents in Long-Term Care: a review of the literature", Journal of Aging and Physical Activity, forthcoming.

[6] Bertrand, M., Luttmer, E. and S. Mullainathan, 2000, "Network effects and welfare cultures", Quarterly Journal of Economics, 115, 1019-1056.

[7] Blain, H., Vuillemin, A., Blain, A. and C. Jeandel, 2000, "Médecine préventive chez les personnes agées. Les effets préventifs de l'activité physique chez les personnes agées", Presse Médicale, 1240-1248.

[8] Carrell, S.E., Hoekstra, M. and J.E. West, 2011, "Is poor fitness contagious?: evidence from randomly assigned friends", Journal of Public Economics, 95, 657663.

[9] Colcombe, S. and A.F. Kramer, 2003, "Fitness effects on the cognitive function of older adults: a meta-analytic study", Psychological Science, 14, 125-130.

[10] Cremer, H., Gahvari, F. and J.M. Lozachmeur, 2010, "Tagging and income taxation: theory and an application", American Economic Journal: Economic Policy, $2,31-50$. 
[11] Eder, D. and S. Parker, 1987, "The cultural production and reproduction of gender: the effect of extracurricular activities on peer-group culture", Sociology of Education, 60, 200-213.

[12] Efrat, M.W., 2009, "The relationship between peer and/or friends? Influence and physical activity among elementary school children: a review", Californian Journal of Health Promotion, 7, 48-61.

[13] Gallagher, K.M. and J.A. Updegraff, 2012, "Health message framing effects on attitudes, intentions, and behavior: a meta-analytic review", Annals of Behavioral Medicine, 43, 101-116.

[14] Glaeser, E., Sacerdote, B. and J. Scheinkman, 1996, "Crime and social interactions", Quarterly Journal of Economics, 111, 507-548.

[15] Hallal, P.C., Andersen, L.B., Bull, F.C., Guthold, R., Haskell, W. and U. Ekelund, 2012, "Global physical activity levels: surveillance progress, pitfalls, and prospects", Lancet, 380, 247-257.

[16] Heath, G.W., Parra, D.C., Sarmiento, O.L., Andersen, L.B., Owen, N., Goenka, S., Montes, F. and R.C. Brownson, 2012, "Evidence-based intervention in physical activity: lessons from around the world", Lancet, 380, 272-281.

[17] Hirvensalo, M. and T. Lintunen, 2011, "Life-course perspective for physical activity and sports participation", European Review of Aging and Physical Activity, 8, 1322.

[18] Katz, M. and C. Shapiro, 1985, Network externalities, competition and compatibility, American Economic Review, 75, 424-440.

[19] Keysor, J.J., 2003, "Does late-life physical activity or exercise prevent or minimize disablement? A critical review of the scientific evidence", American Journal of Preventive Medicine, 25, 129-136. 
[20] Kohl, H.W., Craig, C.L., Lambert, E.V., Inoue, S., Alkandari, J.R., Leetongin, G. and S. Kahlmeier, 2012, "The pandemic of physical inactivity: global action for public health", Lancet, 380, 294-305.

[21] Latimer, A.E., Brawley, L.R. and R.L. Bassett, 2010, "A systematic review of three approaches for constructing physical activity messages: what messages work and what improvements are needed?", International Journal of Behavioral Nutrition and Physical Activity, 7:36.

[22] Lee, I.M., Shiroma, E., Lobelo, F., Puska, P., Blair, S. and P. Katzmarzyk, 2012, "Effect of physical inactivity on major non-communicable diseases worldwide: an analysis of burden of disease and life expectancy", Lancet, 380, 219-229.

[23] McAuley, E., Elavsky, S., Jerome, G.J., Konopack, J.F. and D.X. Marquez, 2005, "Physical activity related well-being in older adults: social cognitive influences", Psychology and Aging, 20, 295-302.

[24] OECD [Organisation for Economic Co-operation and Development], 2010, "Obesity and the economics of prevention: fit not fat", OECD report by F. Sassi.

[25] PAGAC [Physical Activity Guidelines Advisory Committee], 2008, "Physical activity guidelines advisory committee report", Department of Health and Human Services.

[26] Renaud, M. and L. Bherer, 2005, "L'impact de la condition physique sur le vieillissement cognitif", Psychologie \& Neuropsychiatrie du Vieillissement, 3, 199-206.

[27] Robbins, L.B., Stommel, M. and L.M. Hamel, 2008, "Social support for physical activity of middle school students", Public Health Nursing, 25, 451-460.

[28] Sacerdote, B., 2001, "Peer effects with random assignment: results for Darmouth roommates", Quarterly Journal of Economics, 116, 681-704. 
[29] Saez, E. and E. Duflo, 2003, "The role of information and social interactions in retirement plan decisions: evidence from a randomized experiment", Quarterly Journal of Economics, 118, 815-842.

[30] Shephard, R., 1991, "Physical fitness: exercise and ageing" in Principles and practice of geriatric medicine. 2nd Edition. MSJ Pathy John Wiley \& Sons Ttd Ed., 279-292.

[31] Smith, A.L., 2003, "Peer relationships in physical activity contexts: a road less traveled in youth sport and exercise psychology research", Psychology of Sport and Exercise, 4, 25-39.

[32] Trogdon, J., Nonnemaker, J. and J. Pais, 2008, "Peer effects in adolescent overweight", Journal of Health Economics, 27, 1388-1399.

[33] Vogel, T., Brechat, P.-H., Leprï£jtre, P.-M., Kaltenbach, G., Berthel, M. and J. Lonsdorfer, 2009, "Health benefits of physical activity in older patients: a review", International Journal of Clinical Practice, 63, 303-320.

[34] Voorhees, C.C., Murray, D., Welk, G., Birnbaum, A., Ribisl, K. M., Johnson, C.C., Pfeiffer, K.A., Saksvig, B. and J.B. Jobe, 2005, "The role of peer social network factors and physical activity in adolescent girls", American Journal of Health Behavior, 29, 183-190.

[35] Warburton, D.E.R., Nicol, C.W. and S.D. Bredin, 2006, "Health benefits of physical activity: the evidence", Canadian Medical Association Journal, 174, 801-809.

[36] Wechsler, H., Devereaux, R.S., Davis, M. and J. Collins, 2000, "Using the school environment to promote physical activity and healthy eating", Preventive Medicine, 31, S121-S137.

[37] Wen, C.P., Wai, J.P.M., Tsai, M.K., Yang, Y.C., Cheng, T.Y.D., Lee, M-C., Chan, H.T., Tsao, C.K., Tsai, S.P. and X. Wu, 2011, "Minimum amount of physical 
activity for reduced mortality and extended life expectancy: a prospective cohort study", Lancet, 378, 1244-1253.

[38] WHO [World Health Organization], 2010, "Global recommendations on physical activity for health", Geneva: WHO Press, NLM classification: QT 255.

\section{Appendix}

\section{Appendix A - Proof of Proposition 1.}

An LF-equilibrium consists of a pair $\left(\theta_{A}^{L F}, \theta_{B}^{L F}\right) \in(0,1) \times(0,1)$ which solves equations $(2 A),(2 B)$ and $\widehat{\theta}=p \theta_{A}^{L F}+(1-p) \theta_{B}^{L F}$. Solving this system leads to:

$\theta_{A}^{L F}=\frac{4 \alpha_{A} \alpha_{B}-2 \alpha_{B}-\varepsilon(1-p)\left(\alpha_{A}-\alpha_{B}\right)}{2(2-\varepsilon) \alpha_{A} \alpha_{B}} \quad$ and $\quad \theta_{B}^{L F}=\frac{4 \alpha_{A} \alpha_{B}-2 \alpha_{A}+\varepsilon p\left(\alpha_{A}-\alpha_{B}\right)}{2(2-\varepsilon) \alpha_{A} \alpha_{B}}$

Note that $\theta_{A}^{L F}-\theta_{B}^{L F}=\left(\alpha_{A}-\alpha_{B}\right) /\left(2 \alpha_{A} \alpha_{B}\right)$. Therefore, according to Assumption 1 $\theta_{A}^{L F}>\theta_{B}^{L F}$, for all $\varepsilon \in[0,1]$. Consequently, to establish that $\left(\theta_{A}^{L F}, \theta_{B}^{L F}\right) \in(0,1) \times(0,1)$ it is sufficient to show that $\theta_{B}^{L F}>0$ and $\theta_{A}^{L F}<1$. The positivity of $\theta_{B}^{L F}$ is obvious. Having $\theta_{A}^{L F}<1$ is equivalent to $2 \alpha_{B}\left(\varepsilon \alpha_{A}-1\right)-\varepsilon(1-p)\left(\alpha_{A}-\alpha_{B}\right)<0$. Therefore, according to Assumption $1,\left(\theta_{A}^{L F}, \theta_{B}^{L F}\right) \in(0,1) \times(0,1)$ is the LF-equilibrium.

After computations, we obtain $\partial_{\varepsilon} \theta_{A}^{L F}=\partial_{\varepsilon} \theta_{B}^{L F}=\left[2 \alpha_{A} \alpha_{B}-(1-p) \alpha_{A}-p \alpha_{B}\right] /[(2-$ $\left.\varepsilon)^{2} \alpha_{A} \alpha_{B}\right]$. As $\alpha_{B}<\alpha_{A}$ and $2 \alpha_{B}>1,(1-p) \alpha_{A}+p \alpha_{B}<2 \alpha_{A} \alpha_{B}$ and, consequently, $\theta_{A}^{L F}$ and $\theta_{B}^{L F}$ increase in $\varepsilon$. We also have $\partial_{\varepsilon \varepsilon} \theta_{A}^{L F}=\partial_{\varepsilon \varepsilon} \theta_{B}^{L F}=2\left[2 \alpha_{A} \alpha_{B}-(1-p) \alpha_{A}-\right.$ $\left.p \alpha_{B}\right] /\left[(2-\varepsilon)^{3} \alpha_{A} \alpha_{B}\right]$. Then, $\theta_{A}^{L F}$ and $\theta_{B}^{L F}$ are convex functions of $\varepsilon$.

\section{Appendix B - Proof of Proposition 2.}

According to $(3)$ we have $\theta^{G}=\left(1-\widetilde{\varepsilon}_{G}\right) /(1-\varepsilon)$. This value is lower than 1 since, according to Assumption 1, $\widetilde{\varepsilon}_{G}=1 /(2 \Lambda)<1$. Therefore, $\theta^{G}=\left(1-\widetilde{\varepsilon}_{G}\right) /(1-\varepsilon)$ when $\varepsilon \leq \widetilde{\varepsilon}_{G}$, and $\theta^{G}=1$ otherwise.

After computations, $\partial_{\varepsilon} \theta^{G}=[2 \Lambda-1] /\left[2 \Lambda(1-\varepsilon)^{2}\right]$ and $\partial_{\varepsilon \varepsilon} \theta^{G}=[2 \Lambda-1] /\left[\Lambda(1-\varepsilon)^{3}\right]$. As $2 \Lambda>1, \partial_{\varepsilon} \theta^{G}$ and $\partial_{\varepsilon \varepsilon} \theta^{G}$ are positive and $\theta^{G}$ is increasing and convex in $\varepsilon$.

Additionally, $\partial_{\varepsilon \varepsilon} \theta^{G}-\partial_{\varepsilon \varepsilon} \theta_{i}^{L F}$ has the sign of $\alpha_{A} \alpha_{B}\left[(2-\varepsilon)^{3}-2(1-\varepsilon)^{3}\right][2 \Lambda-1]+$ $2 p(1-p)\left(\alpha_{A}-\alpha_{B}\right)^{2}(1-\varepsilon)^{3}$. Since for all $\varepsilon \in[0,1]$ we have $(2-\varepsilon)^{3}>2(1-\varepsilon)^{3}$ and 
$2 \Lambda>1, \partial_{\varepsilon \varepsilon} \theta^{G}-\partial_{\varepsilon \varepsilon} \theta_{i}^{L F}$ is positive and, consequently, $\theta^{G}$ is more convex than $\theta_{A}^{L F}$ and $\theta_{B}^{L F}$

Finally note that $\left.\theta_{A}^{L F}\right|_{\varepsilon=0}=1-1 /\left(2 \alpha_{A}\right)>\left.\theta^{G}\right|_{\varepsilon=0}=1-1 /(2 \Lambda)>\left.\theta_{B}^{L F}\right|_{\varepsilon=0}=$ $1-1 /\left(2 \alpha_{B}\right)$ and, according to Proposition $1,\left.\theta^{G}\right|_{\varepsilon=\widetilde{\varepsilon}_{G}}=1>\left.\theta_{A}^{L F}\right|_{\varepsilon=\widetilde{\varepsilon}_{G}}>\left.\theta_{B}^{L F}\right|_{\varepsilon=\widetilde{\varepsilon}_{G}}$. Therefore, since $\partial_{\varepsilon \varepsilon} \theta_{i}^{L F}<\partial_{\varepsilon \varepsilon} \theta^{G}, \theta_{B}^{L F}<\theta^{G}$ for all $\varepsilon \in\left[0, \widetilde{\varepsilon}_{G}\right]$ and there exists a unique $\bar{\varepsilon}_{G} \in\left(0, \widetilde{\varepsilon}_{G}\right)$ such that $\theta_{A}^{L F} \lessgtr \theta^{G}$ if and only if $\varepsilon \gtrless \bar{\varepsilon}_{G}$.

\section{Appendix C - Proof of Proposition 3 .}

According to $(2 i)(i=A$ or $B)$ we have $\alpha_{i} \varepsilon \widehat{\theta}^{L F}=2 \alpha_{i} \theta_{i}^{L F}-2 \alpha_{i}+1$. Substituting this into (1) allows us to obtain $\mathcal{U}_{i}^{L F}=1+\alpha_{i}\left(\theta_{i}^{L F}\right)^{2}$. Consequently, we have $\partial_{\varepsilon} \mathcal{U}_{i}^{L F}=$ $2 \alpha_{i} \theta_{i}^{L F} \partial_{\varepsilon} \theta_{i}^{L F}$ and $\partial_{\varepsilon \varepsilon} \mathcal{U}_{i}^{L F}=2 \alpha_{i}\left[\left(\partial_{\varepsilon} \theta_{i}^{L F}\right)^{2}+\theta_{i}^{L F} \partial_{\varepsilon \varepsilon} \theta_{i}^{L F}\right]$. Both expressions are positive since $\partial_{\varepsilon} \theta_{i}^{L F}$ and $\partial_{\varepsilon \varepsilon} \theta_{i}^{L F}$ are positive. Therefore, $\mathcal{U}_{A}^{L F}$ and $\mathcal{U}_{B}^{L F}$ are increasing and convex functions of $\varepsilon$.

Substituting (3) into (1) allows us to obtain $\mathcal{U}_{i}^{G}=1+\left[\left(1+\widetilde{\varepsilon}_{G}\right) \alpha_{i}-1\right] \theta^{G}$. Then, we have $\partial_{\varepsilon} \mathcal{U}_{i}^{G}=\left[\left(1+\widetilde{\varepsilon}_{G}\right) \alpha_{i}-1\right] \partial_{\varepsilon} \theta^{G}$ and $\partial_{\varepsilon \varepsilon} \mathcal{U}_{i}^{G}=\left[\left(1+\widetilde{\varepsilon}_{G}\right) \alpha_{i}-1\right] \partial_{\varepsilon \varepsilon} \theta^{G}$. Since $\partial_{\varepsilon} \theta^{G}$ and $\partial_{\varepsilon \varepsilon} \theta^{G}$ are positive, $\partial_{\varepsilon} \mathcal{U}_{i}^{G}$ and $\partial_{\varepsilon \varepsilon} \mathcal{U}_{i}^{G}$ have the sign of $\left(1+\widetilde{\varepsilon}_{G}\right) \alpha_{i}-1$. As $\widetilde{\varepsilon}_{G}>1 /\left(2 \alpha_{A}\right)$ and $\alpha_{A}>1 / 2$ we have $\left(1+\widetilde{\varepsilon}_{G}\right) \alpha_{A}>1$. Consequently, $\mathcal{U}_{A}^{G}$ is an increasing and convex function of $\varepsilon \in\left[0, \widetilde{\varepsilon}_{G}\right)$.

We now contrast $\mathcal{U}_{A}^{L F}$ with $\mathcal{U}_{A}^{G}$. Using Propositions 1 and 2, we obtain $\partial_{\varepsilon} \mathcal{U}_{A}^{G}=$ $\mathcal{A} /\left[4 \Lambda^{2}(1-\varepsilon)^{2}\right]$ and $\partial_{\varepsilon} \mathcal{U}_{A}^{L F}=\mathcal{B} /\left[\alpha_{A} \alpha_{B}^{2}(2-\varepsilon)^{3}\right]$ with $\mathcal{A}=2 \Lambda-\alpha_{A}-4 \Lambda^{2}\left(1-\alpha_{A}\right)$ and $\mathcal{B}=$ $\left[2 \alpha_{B}+\varepsilon(1-p)\left(\alpha_{A}-\alpha_{B}\right)\right]\left[p \alpha_{B}+(1-p) \alpha_{A}\right]-2 \alpha_{A} \alpha_{B}\left[4 \alpha_{B}+(1-p)\left(\alpha_{A}-\alpha_{B}\right)(2+\varepsilon)\right]+8 \alpha_{A}^{2} \alpha_{B}^{2}$.

Then $\partial_{\varepsilon} \mathcal{U}_{A}^{G}-\partial_{\varepsilon} \mathcal{U}_{A}^{L F}$ has the sign of $(2-\varepsilon)^{3} \mathcal{C}-8(1-\varepsilon)^{2} \mathcal{D}$ with $\mathcal{C}=\alpha_{A} \alpha_{B}^{2} \mathcal{A}(\Lambda)$ and $\mathcal{D}=\Lambda^{2} \mathcal{B} / 2$. Using the facts that $\mathcal{C}>0$ and $(2-\varepsilon)^{3} \geq 8(1-\varepsilon)^{2}$, a sufficient condition to show the positivity of $\partial_{\varepsilon} \mathcal{U}_{A}^{G}-\partial_{\varepsilon} \mathcal{U}_{A}^{L F}$ is to establish the positivity of $\mathcal{C}-\mathcal{D}$. Rearranging terms leads to $\mathcal{C}-\mathcal{D}=\left(2 \Lambda-\alpha_{A}\right) \alpha_{A} \alpha_{B}^{2}+\Lambda^{2}\left\{\alpha_{B}\left[(1-p)\left(\alpha_{A}-\alpha_{B}\right)\left(2 \alpha_{A}-1\right)-\alpha_{B}\right]+\varepsilon \mathcal{E}\right\}$ with $2 \mathcal{E}=\left(\alpha_{A}-\alpha_{B}\right)(1-p)\left[\alpha_{B}\left(2 \alpha_{A}-1\right)-(1-p)\left(\alpha_{A}-\alpha_{B}\right)\right]>\left(\alpha_{A}-\alpha_{B}\right)(1-p)\left[\alpha_{A}\left(2 \alpha_{B}-1\right)\right]>$

0 . Let $\sigma=(1-p)\left(\alpha_{A}-\alpha_{B}\right)$. Since $\mathcal{E}>0$ and $\Lambda=\alpha_{A}-\sigma$, we have $\mathcal{C}-\mathcal{D} \geq\left.(\mathcal{C}-\mathcal{D})\right|_{\varepsilon=0}=$ $\alpha_{B}\left(2 \alpha_{A}-1\right) \sigma \mathcal{F}(\sigma)$ with $\mathcal{F}(\sigma)=\sigma^{2}-\left[2 \alpha_{A}+\alpha_{B} /\left(2 \alpha_{A}-1\right)\right] \sigma+\alpha_{A}^{2}$. As $0 \leq \sigma \leq \alpha_{A}-\alpha_{B}$, $\mathcal{F}^{\prime}(\sigma)<0$ and, therefore, $\mathcal{F}(\sigma) \geq \mathcal{F}\left(\alpha_{A}-\alpha_{B}\right)=\alpha_{A} \alpha_{B}\left(2 \alpha_{B}-1\right) /\left(2 \alpha_{A}-1\right) \geq 0$. Consequently, $\mathcal{C}-\mathcal{D}>0$ meaning that $\mathcal{U}_{A}^{G}-\mathcal{U}_{A}^{L F}$ increases in $\varepsilon$ for $\varepsilon \in\left[0, \widetilde{\varepsilon}_{G}\right)$. 
From (1) we obtain $\left.\left(\mathcal{U}_{A}^{G}-\mathcal{U}_{A}^{L F}\right)\right|_{\varepsilon=0}=\left[\left.\theta_{A}^{L F}\right|_{\varepsilon=0}-\left.\theta^{G}\right|_{\varepsilon=0}\right]\left[1+\alpha_{A}\left(\left.\theta_{A}^{L F}\right|_{\varepsilon=0}+\left.\theta^{G}\right|_{\varepsilon=0}-\right.\right.$ 2)]. As $\left.\theta_{A}^{L F}\right|_{\varepsilon=0}=1-1 /\left(2 \alpha_{A}\right)>\left.\theta^{G}\right|_{\varepsilon=0}=1-\widetilde{\varepsilon}_{G}$, we have $\left.\theta_{A}^{L F}\right|_{\varepsilon=0}+\left.\theta^{G}\right|_{\varepsilon=0}<$ $2-1 / \alpha_{A}$. Hence, we obtain $\left.\left(\mathcal{U}_{A}^{G}-\mathcal{U}_{A}^{L F}\right)\right|_{\varepsilon=0}<0$. Given the definition of $\bar{\varepsilon}_{G}$ (see Proposition 2), $\left.\theta_{A}^{L F}\right|_{\varepsilon=\bar{\varepsilon}_{G}}=\left.\theta^{G}\right|_{\varepsilon=\bar{\varepsilon}_{G}} \equiv \bar{\theta}^{L F}$. From (1) it follows that $\left.\left(\mathcal{U}_{A}^{G}-\mathcal{U}_{A}^{L F}\right)\right|_{\varepsilon=\bar{\varepsilon}_{G}}=$ $\alpha_{A} \bar{\varepsilon}_{G} \bar{\theta}^{L F}\left(\bar{\theta}^{L F}-\left.\widehat{\theta}^{L F}\right|_{\varepsilon=\bar{\varepsilon}_{G}}\right)=\alpha_{A} \bar{\varepsilon}_{G} \bar{\theta}^{L F}(1-p)\left(\bar{\theta}^{L F}-\left.\theta_{B}^{L F}\right|_{\varepsilon=\bar{\varepsilon}_{G}}\right)$. Since, according to Proposition 1, $\left.\theta_{B}^{L F}\right|_{\varepsilon=\bar{\varepsilon}_{G}}<\left.\theta_{A}^{L F}\right|_{\varepsilon=\bar{\varepsilon}_{G}}=\bar{\theta}^{L F}$, then $\left.\left(\mathcal{U}_{A}^{G}-\mathcal{U}_{A}^{L F}\right)\right|_{\varepsilon=\bar{\varepsilon}_{G}}>0$. Consequently, since $\mathcal{U}_{A}^{G}-\mathcal{U}_{A}^{L F}$ increases in $\varepsilon$ for $\varepsilon \in\left[0, \widetilde{\varepsilon}_{G}\right)$, there exists a unique $\widehat{\varepsilon}_{A} \in\left(0, \bar{\varepsilon}_{G}\right)$ such that $\mathcal{U}_{A}^{L F}>\mathcal{U}_{A}^{G}$ if $0 \leq \varepsilon<\widehat{\varepsilon}_{A}$ and $\mathcal{U}_{A}^{L F}<\mathcal{U}_{A}^{G}$ if $\widehat{\varepsilon}_{A}<\varepsilon \leq \widetilde{\varepsilon}_{G}$.

\section{Appendix D - Proof of Proposition 4.}

In Appendix $\mathrm{C}$ we establish that $\mathcal{U}_{B}^{L F}$ is an increasing and convex function of $\varepsilon$ and that $\partial_{\varepsilon} \mathcal{U}_{B}^{G}$ and $\partial_{\varepsilon \varepsilon} \mathcal{U}_{B}^{G}$ have the sign of $\left(1+\widetilde{\varepsilon}_{G}\right) \alpha_{B}-1$, i.e., after computations, the sign of $\alpha_{B}-2 \Lambda\left(1-\alpha_{B}\right)=\alpha_{B}\left(2 \alpha_{B}-1\right)-2 p\left(\alpha_{A}-\alpha_{B}\right)\left(1-\alpha_{B}\right)$. Then $\partial_{\varepsilon} \mathcal{U}_{B}^{G}$ and $\partial_{\varepsilon \varepsilon} \mathcal{U}_{B}^{G}$ have the sign of $\widehat{p}-p$ with $\widehat{p}=\min \left\{1, \alpha_{B}\left(2 \alpha_{B}-1\right) /\left[2\left(\alpha_{A}-\alpha_{B}\right)\left(1-\alpha_{B}\right)\right]\right\}$. Consequently, $\mathcal{U}_{B}^{G}$ is a decreasing and concave function of $\varepsilon$ if $p>\widehat{p}$ and an increasing and convex function of $\varepsilon$ if $p \leq \widehat{p}$.

According to (1), $\left.\left(\mathcal{U}_{B}^{G}-\mathcal{U}_{B}^{L F}\right)\right|_{\varepsilon=0}=\left[\left.\theta_{B}^{L F}\right|_{\varepsilon=0}-\left.\theta^{G}\right|_{\varepsilon=0}\right]\left[1+\alpha_{B}\left(\left.\theta_{B}^{L F}\right|_{\varepsilon=0}+\left.\theta^{G}\right|_{\varepsilon=0}-2\right)\right]$. As $\left.\theta_{B}^{L F}\right|_{\varepsilon=0}=1-1 /\left(2 \alpha_{B}\right)<\left.\theta^{G}\right|_{\varepsilon=0}=1-\widetilde{\varepsilon}_{G}$, we have $\left.\theta_{B}^{L F}\right|_{\varepsilon=0}+\left.\theta^{G}\right|_{\varepsilon=0}>2-1 / \alpha_{B}$. Hence, we obtain:

$\Rightarrow$ Fact $1-$ For all $p \in(0,1), \mathcal{U}_{B}^{L F}>\mathcal{U}_{B}^{G}$ when $\varepsilon=0$.

When $p>\widehat{p}, \mathcal{U}_{B}^{L F}$ increases in $\varepsilon$ for $\varepsilon \in\left[0, \widetilde{\varepsilon}_{G}\right]$ while $\mathcal{U}_{B}^{G}$ decreases and, according to Fact 1, we have $\mathcal{U}_{B}^{L F}>\mathcal{U}_{B}^{G}$ when $0 \leq \varepsilon \leq \widetilde{\varepsilon}_{G}$. When $p \leq \widehat{p}$, complications arise since both $\mathcal{U}_{B}^{L F}$ and $\mathcal{U}_{B}^{G}$ increase in $\varepsilon$. According to Appendix $\mathrm{C}, \partial_{\varepsilon} \mathcal{U}_{B}^{L F}-\partial_{\varepsilon} \mathcal{U}_{B}^{G}=$ $2 \alpha_{B} \theta_{B}^{L F} \partial_{\varepsilon} \theta_{B}^{L F}-\left[\left(1+\widetilde{\varepsilon}_{G}\right) \alpha_{B}-1\right] \partial_{\varepsilon} \theta_{B}^{G}$. Then, after computations and using Appendix $\mathrm{A}$ and $\mathrm{B}, \partial_{\varepsilon} \mathcal{U}_{B}^{L F}-\partial_{\varepsilon} \mathcal{U}_{B}^{G}$ has the sign of $\mathcal{P}(\varepsilon)=4 \Lambda^{2}(1-\varepsilon)^{2}\left[4 \alpha_{A} \alpha_{B}-2 \alpha_{A}+\varepsilon p\left(\alpha_{A}-\right.\right.$ $\left.\left.\alpha_{B}\right)\right]\left[2 \alpha_{A} \alpha_{B}-p \alpha_{B}-(1-p) \alpha_{A}\right]-(2-\varepsilon)^{3} \alpha_{A}^{2} \alpha_{B}\left[(1+2 \Lambda) \alpha_{B}-2 \Lambda\right][2 \Lambda-1]$. The degree of this polynomial function is three and $\mathcal{P}(\varepsilon)=8 \Lambda^{2} \alpha_{A}\left[2 \alpha_{B}-1\right]\left[2 \alpha_{A} \alpha_{B}-p \alpha_{B}-(1-p) \alpha_{A}\right]-$ $8 \alpha_{A}^{2} \alpha_{B}\left[(1+2 \Lambda) \alpha_{B}-2 \Lambda\right][2 \Lambda-1]=8 \alpha_{A}\left\{\Lambda^{2} p\left[2 \alpha_{B}-1\right]\left[\alpha_{A}-\alpha_{B}\right]+\alpha_{A}\left[\Lambda-\alpha_{B}\right]^{2}\right\}>0$. Moreover, $\mathcal{P}(1)=-\alpha_{A}^{2} \alpha_{B}\left[(1+2 \Lambda) \alpha_{B}-2 \Lambda\right][2 \Lambda-1]$ and $\mathcal{P}^{\prime}(1)=3 \alpha_{A}^{2} \alpha_{B}\left[(1+2 \Lambda) \alpha_{B}-\right.$ 
$2 \Lambda][2 \Lambda-1]$. As $p \leq \widehat{p},(1+2 \Lambda) \alpha_{B}-2 \Lambda>0$ and consequently $\mathcal{P}(1)<0$ and $\mathcal{P}^{\prime}(1)>0$. The fact that $\mathcal{P}(0)>0, \mathcal{P}(1)<0$ and $\mathcal{P}^{\prime}(1)>0$ implies the existence and the uniqueness of root $\varepsilon_{s}$ between 0 and $1 .{ }^{9}$ Then, $\mathcal{P}(\varepsilon)>0$ if $0 \leq \varepsilon<\varepsilon_{s}$ and $\mathcal{P}(\varepsilon)<0$ if $\varepsilon_{s}<\varepsilon<1$. Consequently, we establish:

$\Rightarrow$ Fact $2-$ When $\varepsilon_{s} \geq \widetilde{\varepsilon}_{G}, \mathcal{U}_{B}^{G}-\mathcal{U}_{B}^{L F}$ decreases in $\varepsilon$ for $\varepsilon \in\left[0, \widetilde{\varepsilon}_{G}\right)$. When $\varepsilon_{s}<\widetilde{\varepsilon}_{G}$, the function $\mathcal{U}_{B}^{G}-\mathcal{U}_{B}^{L F}$ decreases for $\varepsilon \in\left[0, \varepsilon_{s}\right)$ and increases for $\varepsilon \in\left(\varepsilon_{s}, \widetilde{\varepsilon}_{G}\right)$.

Note that $\partial_{p}\left[\left(1+\widetilde{\varepsilon}_{G}\right) \alpha_{B}-1\right]=-\alpha_{B}\left(\alpha_{A}-\alpha_{B}\right) /\left(2 \Lambda^{2}\right), \partial_{p} \theta_{B}^{L F}=\varepsilon\left(\alpha_{A}-\alpha_{B}\right) /[2(2-$ $\left.\varepsilon) \alpha_{A} \alpha_{B}\right]$ and $\partial_{p} \theta^{G}=\left(\alpha_{A}-\alpha_{B}\right) /\left[2 \Lambda^{2}(1-\varepsilon)\right]$. Then $\eta_{1}=\left[\left(1+\widetilde{\varepsilon}_{G}\right) \alpha_{B}-1\right] \partial_{p} \theta^{G}+\theta^{G} \partial_{p}[(1+$ $\left.\left.\widetilde{\varepsilon}_{G}\right) \alpha_{B}-1\right]=-\left[p\left(\alpha_{A}-\alpha_{B}\right)^{2}\right] /\left[2(1-\varepsilon) \Lambda^{3}\right]<0$. As $\mathcal{U}_{B}^{G}-\mathcal{U}_{B}^{L F}=\left[\left(1+\widetilde{\varepsilon}_{G}\right) \alpha_{B}-1\right] \theta^{G}-$ $\alpha_{B}\left(\theta_{B}^{L F}\right)^{2}$, we have $\partial_{p} \mathcal{U}_{B}^{G}-\partial_{p} \mathcal{U}_{B}^{L F}=\eta_{1}-2 \alpha_{B} \theta_{B}^{L F} \partial_{p} \theta_{B}^{L F}<0$. Then, we establish:

$\Rightarrow$ Fact $3-$ The function $\mathcal{U}_{B}^{G}-\mathcal{U}_{B}^{L F}$ is a decreasing function of $p$.

Given $\varepsilon$, the maximum of $\mathcal{U}_{B}^{G}-\mathcal{U}_{B}^{L F}$ is obtained when $p=0$, i.e., $\left.\left(\mathcal{U}_{B}^{G}-\mathcal{U}_{B}^{L F}\right)\right|_{p=0}=$ $\varepsilon^{2}\left(2 \alpha_{B}-1\right)^{2} /\left[4 \alpha_{B}(1-\varepsilon)(2-\varepsilon)^{2}\right]$. Hence:

$\Rightarrow$ Fact $4-$ For all $\varepsilon>0, \mathcal{U}_{B}^{G}-\mathcal{U}_{B}^{L F}>0$ if $p=0$.

Using Fact 1 to Fact 4, it is straightforward to establish the assertion of our proposition using the (possible) existence of two thresholds $\bar{p}$ and $\check{p}(0<\bar{p} \leq \check{p} \leq \widehat{p})$ such that:

If $0<p<\check{p}$, there exists a threshold $\varepsilon_{s}<\widetilde{\varepsilon}_{G}$, such that $\mathcal{U}_{B}^{G}-\mathcal{U}_{B}^{L F}$ decreases in $\varepsilon$ for $\varepsilon \in\left[0, \varepsilon_{s}\right)$, increases in $\varepsilon$ for $\varepsilon \in\left(\varepsilon_{s}, \widetilde{\varepsilon}_{G}\right)$ and:

- If $0<p<\bar{p}$, there exists a unique $\widehat{\varepsilon}_{B} \in\left(\varepsilon_{s}, \widetilde{\varepsilon}_{G}\right)$ such that $\mathcal{U}_{B}^{L F}>\mathcal{U}_{B}^{G}$ when $0 \leq \varepsilon<\widehat{\varepsilon}_{B}$ and $\mathcal{U}_{B}^{L F}<\mathcal{U}_{B}^{G}$ when $\widehat{\varepsilon}_{B}<\varepsilon<\widetilde{\varepsilon}_{G}$.

- If $\bar{p}<p<\check{p}, \mathcal{U}_{B}^{L F}>\mathcal{U}_{B}^{G}$ for all $\varepsilon$.

$\star$ If $\check{p}<p<\widehat{p}, \mathcal{U}_{B}^{G}-\mathcal{U}_{B}^{L F}$ decreases in $\varepsilon$ and $\mathcal{U}_{B}^{L F}>\mathcal{U}^{G}$.

${ }^{9}$ Obviously, $\mathcal{P}(0)>0$ and $\mathcal{P}(1)<0$ guarantee the existence of a root $\varepsilon_{s}$ between 0 and 1 . If $\varepsilon_{s}$ is not the unique root between 0 and 1 , there generally exists three roots $\varepsilon_{1}$, $\varepsilon_{2}$ and $\varepsilon_{3}$ such that $0<\varepsilon_{1}<\varepsilon_{2}<\varepsilon_{3}<1$ and $\mathcal{P}^{\prime}\left(\varepsilon_{1}\right)<0, \mathcal{P}^{\prime}\left(\varepsilon_{2}\right)>0, \mathcal{P}^{\prime}\left(\varepsilon_{3}\right)<0$. Then, as $\mathcal{P}^{\prime}(1)>0, \mathcal{P}^{\prime}(\varepsilon)$ have at least three roots: however this is impossible since $\mathcal{P}^{\prime}(\varepsilon)$ is a polynomial function of degree 2 . Consequently $\varepsilon_{s}$ is unique. 
The threshold $\bar{p}$ is defined, according to Fact 1 to Fact 4, from the value of $p$ such that $\left.\left(\mathcal{U}_{B}^{G}-\mathcal{U}_{B}^{L F}\right)\right|_{\varepsilon=\widetilde{\varepsilon}_{G}}=0$. This value is given by $\left.\alpha_{B}\left(\theta_{B}^{L F}\right)^{2}\right|_{\varepsilon=\widetilde{\varepsilon}_{G}}=\left(1+\widetilde{\varepsilon}_{G}\right) \alpha_{B}-1$ with $\left.\alpha_{B}\left(\theta_{B}^{L F}\right)^{2}\right|_{\varepsilon=\widetilde{\varepsilon}_{G}}=\left[\left(8 \alpha_{A} \alpha_{B}-4 \alpha_{A}+1\right)^{2}\left(\alpha_{A}-\alpha_{B}\right)^{2} p^{2}+8 \alpha_{A} \alpha_{B}\left(2 \alpha_{B}-1\right)\left(8 \alpha_{A} \alpha_{B}-\right.\right.$ $\left.\left.4 \alpha_{A}+1\right)\left(\alpha_{A}-\alpha_{B}\right) p+16 \alpha_{A}^{2} \alpha_{B}^{2}\left(2 \alpha_{B}-1\right)^{2}\right] /\left[64 \alpha_{A}^{2} \alpha_{B}\left(\alpha_{A}-\alpha_{B}\right)^{2} p^{2}+32 \alpha_{A}^{2} \alpha_{B}\left(4 \alpha_{B}-\right.\right.$ 1) $\left.\left(\alpha_{A}-\alpha_{B}\right) p+4 \alpha_{A}^{2} \alpha_{B}\left(4 \alpha_{B}-1\right)^{2}\right]$ and $\left(1+\widetilde{\varepsilon}_{G}\right) \alpha_{B}-1=\left[-2\left(1-\alpha_{B}\right)\left(\alpha_{A}-\alpha_{B}\right) p+\right.$ $\left.\alpha_{B}\left(2 \alpha_{B}-1\right)\right] /\left[2 \alpha_{B}+2\left(\alpha_{A}-\alpha_{B}\right) p\right]$. Then, after computations, $\bar{p}$ is the root of $\mathcal{Q}(p)=$ $B_{1} p^{3}+B_{2} p^{2}+B_{3} p+B_{4}$ with $B_{1}=\left\{8 \alpha_{A}\left[2\left(\alpha_{A}+\alpha_{B}\right)-1\right]+1\right\}\left(\alpha_{A}-\alpha_{B}\right)^{3}, B_{2}=$ $\alpha_{B}\left[16 \alpha_{A}\left(\alpha_{A}+2 \alpha_{B}-1\right)+1\right]\left(\alpha_{A}+\alpha_{B}\right)^{2}, B_{3}=4 \alpha_{A} \alpha_{B}\left[2 \alpha_{B}\left(2 \alpha_{B}-1\right)+\alpha_{A}\left(1-\alpha_{B}\right)\right]\left(\alpha_{A}-\alpha_{B}\right)$ and $B_{4}=-2 \alpha_{A}^{2} \alpha_{B}^{2}\left(2 \alpha_{B}-1\right)$. Since $B_{1}>0, B_{2}>0, B_{3}>0$ and $B_{4}<0$, we have $\mathcal{Q}^{\prime}(p)>0, \mathcal{Q}(0)<0$ and $\lim _{p \rightarrow+\infty} \mathcal{Q}(p)=+\infty$. Then, $\mathcal{Q}(p)$ has a unique root and this latter is positive. Consequently, if $\mathcal{Q}(1)>0$ then this root corresponds to $\bar{p}$ and if $\mathcal{Q}(1)<0$ then $\bar{p}=1$.

By construction, the threshold $\check{p}$ exists only when $\bar{p}<1$ and it corresponds to the unique value $\check{p} \in(0,1)$ such that $\mathcal{P}\left(1 /\left[2 \alpha_{B}+2 \check{p}\left(\alpha_{A}-\alpha_{B}\right)\right]\right)=0$ with $\mathcal{P}($.$) which has$ been defined to prove Fact 2 .

\section{Appendix E - Proof of Corollary 1.}

According to Appendix D, $\widehat{\varepsilon}_{B}>\varepsilon_{s}$ and, consequently, $\widehat{\varepsilon}_{B}$ is on the branch of the function $\mathcal{U}_{B}^{G}-\mathcal{U}_{B}^{L F}$ which increases in $\varepsilon$. Then, as $\mathcal{U}_{B}^{G}-\mathcal{U}_{B}^{L F}$ decreases in $p$ (Fact 3 of Appendix D), the threshold $\widehat{\varepsilon}_{B}$ increases in $p$. Moreover, it is straightforward to establish that $\left.\widehat{\varepsilon}_{B}\right|_{p=0}=0$.

Remark that $\partial_{p}\left[\left(1+\widetilde{\varepsilon}_{G}\right) \alpha_{A}-1\right]=-\left[\alpha_{A}\left(\alpha_{A}-\alpha_{B}\right)\right] /\left[2 \Lambda^{2}\right], \partial_{p} \theta_{A}^{L F}=\varepsilon\left(\alpha_{A}-\alpha_{B}\right) /[2(2-$ $\left.\varepsilon) \alpha_{A} \alpha_{B}\right], \partial_{p} \theta^{G}=\left(\alpha_{A}-\alpha_{B}\right) /\left[2 \Lambda^{2}(1-\varepsilon)\right]$ and $\eta_{2}=\left[\left(1+\widetilde{\varepsilon}_{G}\right) \alpha_{A}-1\right] \partial_{p} \theta^{G}+\theta^{G} \partial_{p}\left[\left(1+\widetilde{\varepsilon}_{G}\right) \alpha_{A}-\right.$ $1]=\left[(1-p)\left(\alpha_{A}-\alpha_{B}\right)^{2}\right] /\left[2(1-\varepsilon) \Lambda^{3}\right]>0 . \operatorname{As}_{\mathcal{A}}^{G}-\mathcal{U}_{A}^{L F}=\left[\left(1+\widetilde{\varepsilon}_{G}\right) \alpha_{A}-1\right] \theta^{G}-\alpha_{A}\left(\theta_{A}^{L F}\right)^{2}$, we have $\partial_{p} \mathcal{U}_{A}^{G}-\partial_{p} \mathcal{U}_{A}^{L F}=\eta_{2}-2 \alpha_{A} \theta_{A}^{L F} \partial_{p} \theta_{A}^{L F}=\left(\alpha_{A}-\alpha_{B}\right)\left\{(1-p)\left(\alpha_{A}-\alpha_{B}\right) /\left[(1-\varepsilon)\left[\alpha_{B}+\right.\right.\right.$ $\left.\left.\left.p\left(\alpha_{A}-\alpha_{B}\right)\right]^{3}\right]-\varepsilon\left[2 \alpha_{B}\left(2 \alpha_{A}-1\right)-\varepsilon(1-p)\left(\alpha_{A}-\alpha_{B}\right)\right] /\left[\alpha_{A} \alpha_{B}^{2}(2-\varepsilon)^{2}\right]\right\} / 2$. Then, it is straightforward to show that $\partial_{p} \mathcal{U}_{A}^{G}-\partial_{p} \mathcal{U}_{A}^{L F}$ is a decreasing function of $p$. Consequently, as $\left.\left(\partial_{p} \mathcal{U}_{A}^{G}-\partial_{p} \mathcal{U}_{A}^{L F}\right)\right|_{p=1}=-\left[\varepsilon\left(\alpha_{A}-\alpha_{B}\right)\left(2 \alpha_{A}-1\right)\right] /\left[\alpha_{A} \alpha_{B}(2-\varepsilon)^{2}\right]$, for all $\varepsilon>0$ there exists a threshold $\widetilde{p} \in[0,1)$ such that $\mathcal{U}_{A}^{G}-\mathcal{U}_{A}^{L F}$ decreases in $p$ if (and only if) $p>\widetilde{p}$. Then, because $\left.\left(\mathcal{U}_{A}^{G}-\mathcal{U}_{A}^{L F}\right)\right|_{p=1}=\left[\left(2 \alpha_{A}-1\right)^{2} \varepsilon^{2}\right] /\left[4 \alpha_{A}(1-\varepsilon)(2-\varepsilon)^{2}\right]>0$ there exists 
a unique $p$ such that $\mathcal{U}_{A}^{G}=\mathcal{U}_{A}^{L F}$, for a given $\varepsilon>0$. Consequently, $\widehat{\varepsilon}_{A}$ is a monotonic function of $p$. As $\left.\widehat{\varepsilon}_{A}\right|_{p=1}=0$, the threshold $\widehat{\varepsilon}_{A}$ decreases in $p$.

To summarize, the threshold $\widehat{\varepsilon}_{B}$ increases in $p$ and $\left.\widehat{\varepsilon}_{B}\right|_{p=0}=0$ while $\widehat{\varepsilon}_{A}$ decreases in $p$ and $\left.\widehat{\varepsilon}_{A}\right|_{p=1}=0$. Then, there exists a unique threshold $p \in(0, \bar{p})$ such that $\widehat{\varepsilon}_{A}$ is larger (resp: lower) than $\widehat{\varepsilon}_{B}$ if and only if $p$ is lower (resp: larger) than $\underline{p}$. Using $\underline{p}$ and Propositions 3 and 4, the assertion of Corollary 1 is straightforward.

\section{Appendix F - Proof of Proposition 5.}

To determine the FB-equilibrium, the government chooses $\theta_{A}^{F B}$ and $\theta_{B}^{F B}$ so that $p \mathcal{U}_{A}+(1-p) \mathcal{U}_{B}$ is maximum given $\widehat{\theta}=p \theta_{A}^{F B}+(1-p) \theta_{B}^{F B}$. We first analyze the case of interior solutions (Step 1) and then consider the possibility of corner solutions (Step 2).

$\Rightarrow$ Step 1 - The case of interior solutions $\theta_{A}^{F B} \in(0,1)$ and $\theta_{B}^{F B} \in(0,1)$.

When FB-equilibrium has interior solutions $\left(\widetilde{\theta}_{A}, \widetilde{\theta}_{B}\right) \in(0,1) \times(0,1)$, the government solves:

$$
\begin{array}{cc}
\max _{\widetilde{\theta}_{A}, \widetilde{\theta}_{B}} & p\left(1-\widetilde{\theta}_{A}\right)+\alpha_{A} p\left[2 \widetilde{\theta}_{A}-\left(\widetilde{\theta}_{A}\right)^{2}\right]+(1-p)\left(1-\widetilde{\theta}_{B}\right)+\alpha_{B}(1-p)\left[2 \widetilde{\theta}_{B}-\left(\widetilde{\theta}_{B}\right)^{2}\right] \\
& +\varepsilon\left[\alpha_{A} p^{2}\left(\widetilde{\theta}_{A}\right)^{2}+\alpha_{B}(1-p)^{2}\left(\widetilde{\theta}_{B}\right)^{2}+\left(\alpha_{A}+\alpha_{B}\right) p(1-p) \widetilde{\theta}_{A} \widetilde{\theta}_{B}\right]
\end{array}
$$

After simplifications, the two FOC are given by:

$$
\begin{aligned}
& 2 \alpha_{A}-1-2 \alpha_{A}(1-p \varepsilon) \widetilde{\theta}_{A}+\varepsilon\left(\alpha_{A}+\alpha_{B}\right)(1-p) \widetilde{\theta}_{B}=0 \\
& 2 \alpha_{B}-1-2 \alpha_{B}[1-(1-p) \varepsilon] \widetilde{\theta}_{B}+\varepsilon\left(\alpha_{A}+\alpha_{B}\right) p \widetilde{\theta}_{A}=0
\end{aligned}
$$

Rearranging terms we obtain:

$$
\widetilde{\theta}_{A}^{F B}=\frac{u_{A}^{F B}}{V} \equiv \frac{2 \alpha_{B}\left(2 \alpha_{A}-1\right)-\varepsilon(1-p)\left(\alpha_{A}-\alpha_{B}\right)\left(1+2 \alpha_{B}\right)}{4 \alpha_{A} \alpha_{B}-4 \alpha_{A} \alpha_{B} \varepsilon-p(1-p)\left(\alpha_{A}-\alpha_{B}\right)^{2} \varepsilon^{2}}
$$

and

$$
\widetilde{\theta}_{B}^{F B}=\frac{u_{B}^{F B}}{V} \equiv \frac{2 \alpha_{A}\left(2 \alpha_{B}-1\right)+\varepsilon p\left(\alpha_{A}-\alpha_{B}\right)\left(1+2 \alpha_{A}\right)}{4 \alpha_{A} \alpha_{B}-4 \alpha_{A} \alpha_{B} \varepsilon-p(1-p)\left(\alpha_{A}-\alpha_{B}\right)^{2} \varepsilon^{2}}
$$

The value $V$ decreases (and is concave) when $\varepsilon$ varies from 0 to 1 . As $\left.V\right|_{\varepsilon=0}<0$ and $\left.V\right|_{\varepsilon=1}>0$, there exists a unique $\bar{\varepsilon}_{V} \in(0,1)$ such that $V$ is positive if and only if 
$\varepsilon \in\left[0, \bar{\varepsilon}_{V}\right)$. As $\varepsilon \in[0,1]$, we have $u_{A}^{F B} \geq\left(2 \alpha_{B}-1\right)\left(\alpha_{A}+\alpha_{B}\right)$. Thus, $u_{A}^{F B}$ is positive and decreases in $\varepsilon$. As $\left.V\right|_{\varepsilon=0}>\left.u_{A}^{F B}\right|_{\varepsilon=0}>0$, there exists a unique $\bar{\varepsilon}_{A} \in\left(0, \bar{\varepsilon}_{V}\right)$ such that $\widetilde{\theta}_{A}^{F B} \in(0,1)$ if and only if $\varepsilon \in\left[0, \bar{\varepsilon}_{A}\right)$. Let $\delta=4 \alpha_{A} \alpha_{B}-(1-p)\left(\alpha_{A}-\alpha_{B}\right)\left(1+2 \alpha_{B}\right)$, then it is straightforward that $\bar{\varepsilon}_{A}=\left[-\delta+\sqrt{\delta^{2}+8 p(1-p)\left(\alpha_{A}-\alpha_{B}\right)^{2} \alpha_{B}}\right] /\left[2 p(1-p)\left(\alpha_{A}-\right.\right.$ $\left.\left.\alpha_{B}\right)^{2}\right]$.

Similarly, it is obvious that $u_{B}^{F B}$ is positive and increases in $\varepsilon$. As $\left.V\right|_{\varepsilon=0}>\left.u_{B}^{F B}\right|_{\varepsilon=0}>$ 0 , there exists a unique $\widetilde{\varepsilon}_{B} \in\left(0, \bar{\varepsilon}_{V}\right)$ such that $\widetilde{\theta}_{B}^{F B} \in(0,1)$ if and only if $\varepsilon \in\left[0, \widetilde{\varepsilon}_{B}\right)$.

After computations, $\partial_{\varepsilon} \widetilde{\theta}_{A}^{F B}=N_{A} / V^{2}$ with $N_{A}=p(1-p)\left(\alpha_{A}-\alpha_{B}\right)^{2} \varepsilon\left[2 \alpha_{B}\left(2 \alpha_{A}-\right.\right.$ $\left.1)+u_{A}^{F B}\right]+\left.4 \alpha_{A} \alpha_{B} u_{A}^{F B}\right|_{\varepsilon=1}$. As $u_{A}^{F B}>0$ and $\left.u_{A}^{F B}\right|_{\varepsilon=1} \geq\left(2 \alpha_{B}-1\right)\left(\alpha_{A}+\alpha_{B}\right)>0, \widetilde{\theta}_{A}^{F B}$ increases in $\varepsilon$.

Moreover, we have $\partial_{\varepsilon \varepsilon} \widetilde{\theta}_{A}^{F B}=\xi_{A} / V^{3}$ with $\xi_{A}=2 p(1-p) u_{A}^{F B}\left(\alpha_{A}-\alpha_{B}\right)^{2} V+2 N_{A}\left[4 \alpha_{A} \alpha_{B}+\right.$ $\left.2 p(1-p)\left(\alpha_{A}-\alpha_{B}\right)^{2}\right]$. As $u_{A}^{F B}$ and $N_{A}$ are positive, $\xi_{A}$ is positive and $\widetilde{\theta}_{A}^{F B}$ is an increasing and convex function of $\varepsilon$ when $\varepsilon \in\left[0, \bar{\varepsilon}_{A}\right)$. Note that $\left.\widetilde{\theta}_{A}^{F B}\right|_{\varepsilon=0}=\left(2 \alpha_{A}-1\right) /\left(2 \alpha_{A}\right)=$ $\left.\widetilde{\theta}_{A}^{L F}\right|_{\varepsilon=0}$ and $\left.\widetilde{\theta}_{A}^{F B}\right|_{\varepsilon=\bar{\varepsilon}_{A}}=1$.

We follow by contrasting $\left(\widetilde{\theta}_{A}^{F B}, \widetilde{\theta}_{B}^{F B}\right)$ with $\left(\theta_{A}^{L F}, \theta_{B}^{L F}\right)$ and $\theta^{G}$. Note that $\widetilde{\theta}_{A}^{F B}-\theta_{A}^{L F}$ has the sign of $4 \alpha_{A} \alpha_{B} u_{A}^{F B}(1-\varepsilon / 2)-\left[2 \alpha_{B}\left(2 \alpha_{A}-1\right)-\varepsilon(1-p)\left(\alpha_{A}-\alpha_{B}\right)\right] V$, i.e., the sign of $2 \alpha_{A} \alpha_{B} \varepsilon\left[2 \alpha_{B}\left(2 \alpha_{A}-1\right)-(1-p)\left(\alpha_{A}-\alpha_{B}\right)\left\{\varepsilon+2 \alpha_{B}(2-\varepsilon)\right\}\right]+\varepsilon^{2} p(1-p)\left(\alpha_{A}-\right.$ $\left.\alpha_{B}\right)^{2}\left[2 \alpha_{B}\left(2 \alpha_{A}-1\right)-\varepsilon(1-p)\left(\alpha_{A}-\alpha_{B}\right)\right]$. As $2 \alpha_{B}\left(2 \alpha_{A}-1\right)-(1-p)\left(\alpha_{A}-\alpha_{B}\right)[\varepsilon+$ $\left.2 \alpha_{B}(2-\varepsilon)\right] \geq\left(2 \alpha_{B}-1\right)\left[2 \alpha_{B}+\varepsilon\left(\alpha_{A}-\alpha_{B}\right)\right]>0, \widetilde{\theta}_{A}^{F B}-\theta_{A}^{L F}$ is positive, i.e., $\widetilde{\theta}_{A}^{F B} \geq \theta_{A}^{L F}$.

Moreover, $\widetilde{\theta}_{A}^{F B}-\theta^{G}$ has the sign of $\left[2 \alpha_{B}\left(2 \alpha_{A}-1\right)-\varepsilon(1-p)\left(\alpha_{A}-\alpha_{B}\right)\left(1+2 \alpha_{B}\right)\right] 2(1-$ $\varepsilon) \Lambda-(2 \Lambda-1)\left[4 \alpha_{A} \alpha_{B}-4 \alpha_{A} \alpha_{B} \varepsilon-p(1-p)\left(\alpha_{A}-\alpha_{B}\right)^{2} \varepsilon^{2}\right]$, i.e., the sign of $\mathcal{P}_{A}(\varepsilon)=$ $(1-p)\left(\alpha_{A}-\alpha_{B}\right)\left[2 \Lambda\left(1+2 \alpha_{B}\right)+(2 \Lambda-1) p\left(\alpha_{A}-\alpha_{B}\right)\right] \varepsilon^{2}-\left[4 \alpha_{B}\left(\alpha_{A}-\Lambda\right)+2 \Lambda(1-p)\left(\alpha_{A}-\right.\right.$ $\left.\left.\alpha_{B}\right)\left(1+2 \alpha_{B}\right)\right] \varepsilon+4 \alpha_{B}\left(\alpha_{A}-\Lambda\right)$. As $\alpha_{A}-\Lambda=(1-p)\left(\alpha_{A}-\alpha_{B}\right)$ and $p\left(\alpha_{A}-\alpha_{B}\right)=\Lambda-\alpha_{B}$ we obtain after computations $\mathcal{P}_{A}(\varepsilon)=(1-p)\left(\alpha_{A}-\alpha_{B}\right) \mathcal{Q}_{A}(\varepsilon)$ with $\mathcal{Q}_{A}(\varepsilon)=\left[2 \Lambda^{2}+\right.$ $\left.\left(1+2 \alpha_{B}\right) \Lambda+\alpha_{B}\right] \varepsilon^{2}-\left[2\left(1+2 \alpha_{B}\right) \Lambda+4 \alpha_{B}\right] \varepsilon+4 \alpha_{B}$. Then, the discriminant $\Delta_{A}$ of the polynomial function $\mathcal{Q}_{A}(\varepsilon)$ is such that $\Delta_{A}=4 \Lambda^{2}\left(2 \alpha_{B}-1\right)^{2}$. Consequently, the lowest root of $\mathcal{P}_{A}(\varepsilon)$ is $\check{\varepsilon}_{A}=\left[2\left(\alpha_{B}+\Lambda\right)\right] /\left[2 \Lambda^{2}+\left(1+2 \alpha_{B}\right) \Lambda+\alpha_{B}\right]$. As $\mathcal{P}_{A}(0)>0$ and $\check{\varepsilon}_{A}>1$, we have $\mathcal{P}_{A}(\varepsilon)>0$ for all $\varepsilon \in[0,1]$ and, consequently, $\widetilde{\theta}_{A}^{F B}>\theta^{G}$.

Note that $\partial_{\varepsilon} \widetilde{\theta}_{B}^{F B}=N_{B} / V^{2}>0$ with $N_{B}=p^{2}(1-p)\left(\alpha_{A}-\alpha_{B}\right)^{3}\left(1+2 \alpha_{A}\right) \varepsilon^{2}+4 \alpha_{A} p(1-$ 
$p)\left(\alpha_{A}-\alpha_{B}\right)^{2}\left(2 \alpha_{B}-1\right) \varepsilon+\left.4 \alpha_{A} \alpha_{B} u_{B}^{F B}\right|_{\varepsilon=1}$. Therefore $\widetilde{\theta}_{B}^{F B}$ increases in $\varepsilon$. Moreover, as $N_{B}$ and $\partial_{\varepsilon} N_{B}$ are positive, and $\partial_{\varepsilon} V$ is negative, $\widetilde{\theta}_{B}^{F B}$ is a convex function of $\varepsilon$ if $\varepsilon \in\left[0, \widetilde{\varepsilon}_{B}\right)$. Remark that $\left.\widetilde{\theta}_{B}^{F B}\right|_{\varepsilon=0}=\left(2 \alpha_{B}-1\right) /\left(2 \alpha_{B}\right)=\left.\theta_{B}^{L F}\right|_{\varepsilon=0}$ and $\left.\widetilde{\theta}_{B}^{F B}\right|_{\varepsilon=\widetilde{\varepsilon}_{B}}=1$. As the numerator of $\widetilde{\theta}_{B}^{F B}$ is larger than the one of $\theta_{B}^{L F}$ whereas the denominator of $\widetilde{\theta}_{B}^{F B}$ is lower than the one of $\theta_{B}^{L F}$, we have $\widetilde{\theta}_{B}^{F B} \geq \theta_{B}^{L F}$.

Finally, $\theta^{G}-\widetilde{\theta}_{B}^{F B}$ has the sign of $(2 \Lambda-1)\left[4 \alpha_{A} \alpha_{B}-4 \alpha_{A} \alpha_{B} \varepsilon-p(1-p)\left(\alpha_{A}-\alpha_{B}\right)^{2} \varepsilon^{2}\right]-$ $2 \Lambda(1-\varepsilon)\left[2 \alpha_{A}\left(2 \alpha_{B}-1\right)+\varepsilon p\left(\alpha_{A}-\alpha_{B}\right)\left(1+2 \alpha_{A}\right)\right]$, i.e., the sign of $\mathcal{P}_{B}(\varepsilon)=p\left(\alpha_{A}-\right.$ $\left.\alpha_{B}\right)\left[2 \Lambda\left\{1+2 \alpha_{A}-(1-p)\left(\alpha_{A}-\alpha_{B}\right)\right\}+(1-p)\left(\alpha_{A}-\alpha_{B}\right)\right] \varepsilon^{2}-\left[4 \alpha_{A}\left(\Lambda-\alpha_{B}\right)+2 p \Lambda\left(\alpha_{A}-\right.\right.$ $\left.\left.\alpha_{B}\right)\left(1+2 \alpha_{A}\right)\right] \varepsilon+4 \alpha_{A}\left(\Lambda-\alpha_{B}\right)$. As $\Lambda-\alpha_{B}=p\left(\alpha_{A}-\alpha_{B}\right)$ and $(1-p)\left(\alpha_{A}-\alpha_{B}\right)=\alpha_{A}-\Lambda$ we obtain after computations $\mathcal{P}_{B}(\varepsilon)=p\left(\alpha_{A}-\alpha_{B}\right) \mathcal{Q}_{B}(\varepsilon)$ with $\mathcal{Q}_{B}(\varepsilon)=\left[2 \Lambda^{2}+(1+\right.$ $\left.\left.2 \alpha_{A}\right) \Lambda+\alpha_{A}\right] \varepsilon^{2}-\left[2\left(1+2 \alpha_{A}\right) \Lambda+4 \alpha_{A}\right] \varepsilon+4 \alpha_{A}$. Then, the discriminant $\Delta_{B}$ of the polynomial function $\mathcal{Q}_{B}(\varepsilon)$ is such that $\Delta_{B}=4 \Lambda^{2}\left(2 \alpha_{A}-1\right)^{2}$. Consequently, the lowest root of $\mathcal{P}_{B}(\varepsilon)$ is $\check{\varepsilon}_{B}=\left[2\left(\alpha_{A}+\Lambda\right)\right] /\left[2 \Lambda^{2}+\left(1+2 \alpha_{A}\right) \Lambda+\alpha_{A}\right]$. As $\mathcal{P}_{B}(0)>0$ and $\check{\varepsilon}_{B}>1$, we have $\mathcal{P}_{B}(\varepsilon)>0$ for all $\varepsilon \in[0,1]$ and, consequently, $\theta^{G}>\widetilde{\theta}_{B}^{F B}$.

As regards the comparison between $\widetilde{\theta}_{A}^{F B}$ and $\widetilde{\theta}_{B}^{F B}$, when $\varepsilon<\bar{\varepsilon}_{V}, \widetilde{\theta}_{A}^{F B}-\widetilde{\theta}_{B}^{F B}$ has the sign of $u_{A}^{F B}-u_{B}^{F B}$, i.e. the sign of $2-\varepsilon(1+2 \Lambda)$. Then, $\widetilde{\theta}_{A}^{F B}-\widetilde{\theta}_{B}^{F B}$ decreases in $\varepsilon$. Consequently, $\widetilde{\theta}_{A}^{F B}>\widetilde{\theta}_{B}^{F B}$ if and only if $\varepsilon<\widehat{\varepsilon}=2 /(1+2 \Lambda)$. After computations:

$\left.\left(u_{B}^{F B}-V\right)\right|_{\varepsilon=\widehat{\varepsilon}}=\frac{2 \alpha_{A}\left(4 \alpha_{B}^{2}-1\right)+4 p\left(\alpha_{A}-\alpha_{B}\right)^{2}+2 p\left(\alpha_{A}-\alpha_{B}\right)\left[2 \alpha_{A}\left(2 \alpha_{B}-1\right)+2 \alpha_{B}+1\right]}{(1+2 \Lambda)^{2}}$

The positivity of this quantity implies that $\widetilde{\theta}_{A}^{F B}=\widetilde{\theta}_{B}^{F B}$ arises for a $\tilde{\theta}$ larger than 1 . Hence, $\bar{\varepsilon}_{A}<\widetilde{\varepsilon}_{B}<1$ and $\widetilde{\theta}_{A}^{F B}>\widetilde{\theta}_{B}^{F B}$ for all $\varepsilon \in\left[0, \widetilde{\varepsilon}_{B}\right)$.

$\Rightarrow$ Step $2-$ The case of corner solutions $\theta_{A}^{F B}=1$ and $\theta_{B}^{F B} \in(0,1)$.

When $\varepsilon>\bar{\varepsilon}_{A}, \theta_{A}^{F B}=1$ and the value $\bar{\theta}_{B}^{F B}$ which defines $\theta_{B}^{F B}$ solves:

$$
\begin{array}{ll}
\max _{\bar{\theta}_{B}} & \alpha_{A} p+(1-p)\left(1-\bar{\theta}_{B}\right)+\alpha_{B}(1-p)\left[2 \bar{\theta}_{B}-\left(\bar{\theta}_{B}\right)^{2}\right] \\
& +\varepsilon\left[\alpha_{A} p^{2}+\alpha_{B}(1-p)^{2}\left(\bar{\theta}_{B}\right)^{2}+\left(\alpha_{A}+\alpha_{B}\right) p(1-p) \bar{\theta}_{B}\right]
\end{array}
$$

Then, the FOC is $2 \alpha_{B}-1-2 \alpha_{B}[1-\varepsilon(1-p)] \bar{\theta}_{B}+\varepsilon p\left(\alpha_{A}+\alpha_{B}\right)=0$ and:

$$
\bar{\theta}_{B}^{F B}=\frac{2 \alpha_{B}-1+\varepsilon p\left(\alpha_{A}+\alpha_{B}\right)}{2 \alpha_{B}[1-\varepsilon(1-p)]}
$$

which is lower than 1 if and only if $\varepsilon<\bar{\varepsilon}_{B}=1 /\left(\alpha_{B}+\Lambda\right)$. 
After computations $\partial_{\varepsilon} \bar{\theta}_{B}^{F B}=\left[2 \alpha_{B}-1+p\left(1+\alpha_{A}-\alpha_{B}\right)\right] /\left[2 \alpha_{B}[1-\varepsilon(1-p)]^{2}\right]$ and $\partial_{\varepsilon \varepsilon} \bar{\theta}_{B}^{F B}=\left\{(1-p)\left[2 \alpha_{B}-1+p\left(1+\alpha_{A}-\alpha_{B}\right)\right]\right\} /\left\{\alpha_{B}[1-\varepsilon(1-p)]^{3}\right\}$. Then, $\bar{\theta}_{B}^{F B}$ is an increasing and convex function of $\varepsilon$.

Comparing $\theta^{G}$ with $\bar{\theta}_{B}^{F B}$ we conclude that $\theta^{G}-\bar{\theta}_{B}^{F B}$ has the sign of $(2 \Lambda-1) 2 \alpha_{B}[1-$ $\varepsilon(1-p)]-2 \Lambda(1-\varepsilon)\left[2 \alpha_{B}-1+p \varepsilon\left(\alpha_{A}+\alpha_{B}\right)\right]$, i.e., the sign of $\mathcal{R}_{B}(\varepsilon)=2 p\left(\alpha_{A}+\right.$ $\left.\alpha_{B}\right) \Lambda \varepsilon^{2}-2\left[p\left(\alpha_{A}-\alpha_{B}\right) \Lambda+\Lambda-\alpha_{B}+p \alpha_{B}\right] \varepsilon+2\left(\Lambda-\alpha_{B}\right)$. As $\Lambda-\alpha_{B}=p\left(\alpha_{A}-\alpha_{B}\right)$ we obtain $\mathcal{R}_{B}(\varepsilon)=2 p \mathcal{S}_{B}(\varepsilon)$ with $\mathcal{S}_{B}(\varepsilon)=\left(\alpha_{A}+\alpha_{B}\right) \Lambda \varepsilon^{2}-\left[\alpha_{A}+\left(\alpha_{A}-\alpha_{B}\right) \Lambda\right] \varepsilon+\alpha_{A}-\alpha_{B}$. As the discriminant $\Delta_{S}$ of $\mathcal{S}_{B}(\varepsilon)$ is $\Delta_{S}=\left[\alpha_{A}-\left(\alpha_{A}-\alpha_{B}\right) \Lambda\right]^{2}+4 \Lambda\left(\alpha_{A}+\alpha_{B}\right) \alpha_{B}$, the polynomial function $\mathcal{R}_{B}(\varepsilon)$ has two positive roots and the product of these roots is given by $\pi_{R}=\Lambda\left(\alpha_{A}+\alpha_{B}\right) /\left(\alpha_{A}-\alpha_{B}\right)$. As $\pi_{R} \geq \alpha_{B}\left(\alpha_{A}+\alpha_{B}\right) /\left(\alpha_{A}-\alpha_{B}\right) \geq$ $0.5\left(\alpha_{A}+0.5\right) /\left(\alpha_{A}-0.5\right) \geq \alpha_{A}+0.5 \geq 1, \mathcal{R}_{B}(0)>0$ and $\mathcal{R}_{B}(1)>0$, the two roots of $\mathcal{R}_{B}(\varepsilon)$ are larger than 1 and $\mathcal{R}_{B}$ is positive for all $\varepsilon \in(0,1)$. Consequently, $\theta^{G}>\bar{\theta}_{B}^{F B}$.

\section{$\Rightarrow$ Step 3 -Characterization and properties of the FB-equilibrium.}

Using Step 1 and 2, it is straightforward to obtain that the FB-equilibrium $\left(\theta_{A}^{F B}, \theta_{B}^{F B}\right) \in$ $(0,1] \times(0,1]$ is given by:

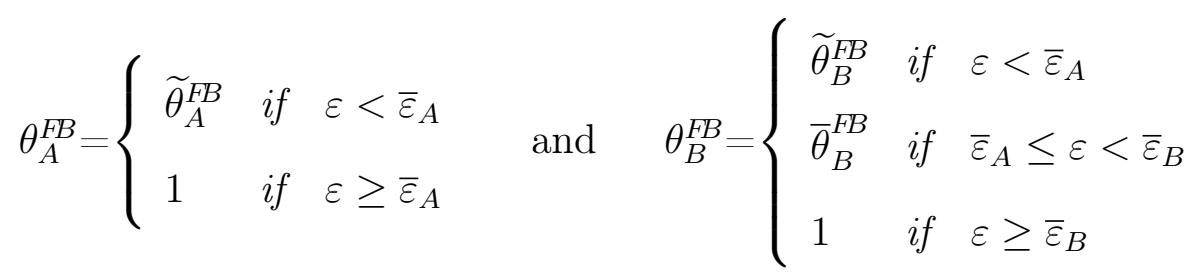

In Step 1 we have established that $\widetilde{\theta}_{A}^{F B}$ and $\widetilde{\theta}_{B}^{F B}$ are both increasing and convex functions of $\varepsilon$. In Step 2 we have established that $\bar{\theta}_{B}^{F B}$ is an increasing and convex function of $\varepsilon$. Combining Step 1 and Step 2 we have also established that $\theta_{A}^{F B}>\theta_{B}^{F B}$, $\theta_{A}^{F B} \geq \theta_{A}^{L F}, \theta_{B}^{F B} \geq \theta_{B}^{L F}, \theta^{G}<\theta_{A}^{F B}$ and $\theta^{G}>\theta_{B}^{F B}$

\section{Appendix G - Proof of Proposition 6 .}

We separately study the welfare of each type of individual.

$\Rightarrow$ Step 1 - Characterization of type $A$ individuals' welfare at the $\mathbf{F B}-$ equilibrium.

We first focus on the welfare of type $A$ individuals when $\varepsilon<\bar{\varepsilon}_{A}$. Merging (6) and (7) in Appendix F gives $\varepsilon\left(\alpha_{A}+\alpha_{B}\right) \widehat{\theta}=2-2 \alpha_{A}-2 \alpha_{B}+2 \alpha_{A}(1-p \varepsilon) \widetilde{\theta}_{A}+2 \alpha_{B}[1-(1-p) \varepsilon] \widetilde{\theta}_{B}$. 
Then, according to $(7)$ and using the fact that $2 \alpha_{B}[1-(1-p) \varepsilon] \widetilde{\theta}_{B}=2 \alpha_{B}-1+\varepsilon\left(\alpha_{A}+\right.$ $\left.\alpha_{B}\right) p \widetilde{\theta}_{A}$ we obtain $\left(\alpha_{A}+\alpha_{B}\right) \varepsilon \widehat{\theta}=\left[2 \alpha_{A}-\varepsilon p\left(\alpha_{A}-\alpha_{B}\right)\right] \widetilde{\theta}_{A}-\left(2 \alpha_{A}-1\right)$. Thus, the utility $\mathcal{U}_{A}^{F B}=1-\widetilde{\theta}_{A}+\alpha_{A}\left[2 \widetilde{\theta}_{A}-\widetilde{\theta}_{A}^{2}+\varepsilon \widetilde{\theta}_{A} \widehat{\theta}\right]$ becomes:

$$
\widetilde{\mathcal{U}}_{A}^{F B}=1+\left(\frac{\alpha_{B}\left(2 \alpha_{A}-1\right)}{\alpha_{A}+\alpha_{B}}\right) \widetilde{\theta}_{A}^{F B}+\left(\frac{\alpha_{A}\left(\alpha_{A}-\alpha_{B}\right)(1-\varepsilon p)}{\alpha_{A}+\alpha_{B}}\right)\left(\widetilde{\theta}_{A}^{F B}\right)^{2}
$$

Then, $\widetilde{\mathcal{U}}_{A}^{F B}=1+G(\varepsilon) /\left(\alpha_{A}+\alpha_{B}\right)$ with $G(\varepsilon)=\alpha_{B}\left(2 \alpha_{A}-1\right) \widetilde{\theta}_{A}^{F B}+\alpha_{A}\left(\alpha_{A}-\alpha_{B}\right)(1-$ $\varepsilon p)\left(\widetilde{\theta}_{A}^{F B}\right)^{2}$. We obtain $G^{\prime}(\varepsilon)=\vartheta(\varepsilon)+2 \alpha_{A}\left(\alpha_{A}-\alpha_{B}\right)(1-\varepsilon p) \widetilde{\theta}_{A}^{F B} \partial_{\varepsilon} \widetilde{\theta}_{A}^{F B}$ with $\vartheta(\varepsilon)=$ $\alpha_{B}\left(2 \alpha_{A}-1\right) \partial_{\varepsilon} \widetilde{\theta}_{A}^{F B}-\alpha_{A} p\left(\alpha_{A}-\alpha_{B}\right)\left(\widetilde{\theta}_{A}^{F B}\right)^{2}$. Then, $\vartheta(\varepsilon)=\left[\alpha_{B}\left(2 \alpha_{A}-1\right) N_{A}-\alpha_{A} p\left(\alpha_{A}-\right.\right.$ $\left.\left.\alpha_{B}\right)\left(u_{A}^{F B}\right)^{2}\right] / V^{2}$. As $N_{A} \geq\left. 4 \alpha_{A} \alpha_{B} u_{A}^{F B}\right|_{\varepsilon=1}$ and $u_{A}^{F B} \leq 2 \alpha_{B}\left(2 \alpha_{A}-1\right)$ we have $\vartheta(\varepsilon) \geq$ $4 \alpha_{A} \alpha_{B}^{2}\left(2 \alpha_{A}-1\right) \Pi / V^{2}$, with $\Pi=\left.u_{A}^{F B}\right|_{\varepsilon=1}-p\left(\alpha_{A}-\alpha_{B}\right)\left(2 \alpha_{A}-1\right)=\left(2 \alpha_{B}-1\right)\left(\alpha_{A}+\right.$ $\left.\alpha_{B}\right)+2 p\left(\alpha_{A}-\alpha_{B}\right)\left[1-\left(\alpha_{A}-\alpha_{B}\right)\right]$. Then $\vartheta(\varepsilon) \geq 0$. This implies $G^{\prime}(\varepsilon)>0$. As $\partial_{\varepsilon} \widetilde{\mathcal{U}}_{A}^{F B}=G^{\prime}(\varepsilon) /\left(\alpha_{A}+\alpha_{B}\right), \widetilde{\mathcal{U}}_{A}^{F B}$ increases in $\varepsilon$.

Moreover $G^{\prime \prime}(\varepsilon)=2 \alpha_{A}\left(\alpha_{A}-\alpha_{B}\right)(1-\varepsilon p)\left[\widetilde{\theta}_{A}^{F B} \partial_{\varepsilon \varepsilon} \widetilde{\theta}_{A}^{F B}+\left(\partial_{\varepsilon} \widetilde{\theta}_{A}^{F B}\right)^{2}\right]+\Delta(\varepsilon)$ with $\Delta(\varepsilon)=$ $\alpha_{B}\left(2 \alpha_{A}-1\right) \partial_{\varepsilon \varepsilon} \widetilde{\theta}_{A}^{F B}-4 \alpha_{A}\left(\alpha_{A}-\alpha_{B}\right) p \widetilde{\theta}_{A}^{F B} \partial_{\varepsilon} \widetilde{\theta}_{A}^{F B}$. Then $\Delta(\varepsilon)=\delta_{A} / V^{3}$ with $\delta_{A}=\alpha_{B}\left(2 \alpha_{A}-\right.$ 1) $\xi_{A}-4 \alpha_{A}\left(\alpha_{A}-\alpha_{B}\right) p N_{A} u_{A}^{F B}$. As $u_{A}^{F B} \leq 2 \alpha_{B}\left(2 \alpha_{A}-1\right)$, we have $\delta_{A} \geq 2 \alpha_{B}\left(2 \alpha_{A}-1\right) p(1-$ p) $u_{A}^{F B}\left(\alpha_{A}-\alpha_{B}\right)^{2} V+4 N_{A} \alpha_{B}\left(2 \alpha_{A}-1\right)\left[2 \alpha_{A} \alpha_{B}-p\left(\alpha_{A}-\alpha_{B}\right)\left\{\alpha_{A}+\alpha_{B}+p\left(\alpha_{A}-\alpha_{B}\right)\right\}\right] \geq$ $2 \alpha_{B}\left(2 \alpha_{A}-1\right) p(1-p) u_{A}^{F B}\left(\alpha_{A}-\alpha_{B}\right)^{2} V+8 N_{A} \alpha_{A} \alpha_{B}\left(2 \alpha_{A}-1\right)\left(2 \alpha_{B}-\alpha_{A}\right)>0$. Then, $G^{\prime \prime}(\varepsilon)$ is positive. As $\partial_{\varepsilon \varepsilon} \widetilde{\mathcal{U}}_{A}^{F B}=G^{\prime \prime}(\varepsilon) /\left(\alpha_{A}+\alpha_{B}\right), \widetilde{\mathcal{U}}_{A}^{F B}$ is a convex function of $\varepsilon$.

We now focus on type $A$ individuals' welfare when $\bar{\varepsilon}_{A} \leq \varepsilon<\widetilde{\varepsilon}_{G}$. As $\theta_{A}^{F B}=1$, then $\overline{\mathcal{U}}_{A}^{F B}=\alpha_{A}(1+\varepsilon \widehat{\theta})$, with $\widehat{\theta}=p+(1-p) \bar{\theta}_{B}^{F B}$. Thus, $\overline{\mathcal{U}}_{A}^{F B}=\alpha_{A}\left[1+\varepsilon p+(1-p) \varepsilon \bar{\theta}_{B}^{F B}\right]$. As $\bar{\theta}_{B}^{F B}$ is a positive, increasing and convex function of $\varepsilon$, it is straightforward to establish that $\overline{\mathcal{U}}_{A}^{F B}$ is an increasing and convex function of $\varepsilon$.

$\Rightarrow$ Step 2 - Characterization of type $B$ individuals' welfare at the FBequilibrium.

We now focus on type $B$ individuals' welfare when $\varepsilon<\bar{\varepsilon}_{A}$. Merging (6) and (7) in Appendix F gives $\varepsilon\left(\alpha_{A}+\alpha_{B}\right) \widehat{\theta}=2-2 \alpha_{A}-2 \alpha_{B}+2 \alpha_{A}(1-p \varepsilon) \widetilde{\theta}_{A}+2 \alpha_{B}[1-(1-p) \varepsilon] \widetilde{\theta}_{B}$. Then, according to (6) and using the fact that $2 \alpha_{A}(1-p \varepsilon) \widetilde{\theta}_{A}=2 \alpha_{A}-1+\varepsilon\left(\alpha_{A}+\right.$ $\left.\alpha_{B}\right)(1-p) \widetilde{\theta}_{B}$ we obtain $\left(\alpha_{A}+\alpha_{B}\right) \varepsilon \widehat{\theta}=\left[2 \alpha_{B}+\varepsilon(1-p)\left(\alpha_{A}-\alpha_{B}\right)\right] \widetilde{\theta}_{B}-\left(2 \alpha_{B}-1\right)$. Then, the utility $\mathcal{U}_{B}^{F B}=1-\widetilde{\theta}_{B}+\alpha_{B}\left[2 \widetilde{\theta}_{B}-\widetilde{\theta}_{B}^{2}+\varepsilon \widetilde{\theta}_{B} \widehat{\theta}\right]$ becomes: 


$$
\widetilde{\mathcal{U}}_{B}^{F B}=1+\left(\frac{\alpha_{A}\left(2 \alpha_{B}-1\right)}{\alpha_{A}+\alpha_{B}}\right) \widetilde{\theta}_{B}^{F B}-\left(\frac{\alpha_{B}\left(\alpha_{A}-\alpha_{B}\right)[1-\varepsilon(1-p)]}{\alpha_{A}+\alpha_{B}}\right)\left(\widetilde{\theta}_{B}^{F B}\right)^{2}
$$

Then, $\widetilde{\mathcal{U}}_{B}^{F B}=1+H(\varepsilon) /\left(\alpha_{A}+\alpha_{B}\right)$ with $H(\varepsilon)=\alpha_{A}\left(2 \alpha_{B}-1\right) \widetilde{\theta}_{B}^{F B}-\alpha_{B}\left(\alpha_{A}-\alpha_{B}\right)[1-$ $\varepsilon(1-p)]\left(\widetilde{\theta}_{B}^{F B}\right)^{2}$. After computations, $H^{\prime}(\varepsilon)=\alpha_{A}\left(2 \alpha_{B}-1\right) \partial_{\varepsilon} \widetilde{\theta}_{B}^{F B}+\alpha_{B}\left(\alpha_{A}-\alpha_{B}\right)(1-$ $p)\left(\widetilde{\theta}_{B}^{F B}\right)^{2}-2[1-\varepsilon(1-p)] \alpha_{B}\left(\alpha_{A}-\alpha_{B}\right) \widetilde{\theta}_{B}^{F B} \partial_{\varepsilon} \widetilde{\theta}_{B}^{F B}$. As $\left.\widetilde{\theta}_{B}^{F B}\right|_{\varepsilon=0}=\left.\partial_{\varepsilon} \widetilde{\theta}_{B}^{F B}\right|_{\varepsilon=0}=\left(2 \alpha_{B}-\right.$ 1) $/\left(2 \alpha_{B}\right)$, we obtain after simplifying $H^{\prime}(0)=\left(2 \alpha_{B}-1\right)\left[2 \alpha_{B}-1\right]\left[2 \alpha_{A}-\left(\alpha_{A}-\alpha_{B}\right)(1+\right.$ $p)] /\left(4 \alpha_{B}\right)$. Therefore, $H^{\prime}(0)>0$ and, by continuity, $\widetilde{\mathcal{U}}_{B}^{F B}$ is an increasing function for sufficiently low values of $\varepsilon$.

We now focus on type $B$ individuals' welfare when $\bar{\varepsilon}_{A} \leq \varepsilon<\widetilde{\varepsilon}_{G}$. Using the fact that $\mathcal{U}_{B}^{F B}=1-\bar{\theta}_{B}^{F B}+\alpha_{B}\left[2 \bar{\theta}_{B}^{F B}-\left(\bar{\theta}_{B}^{F B}\right)^{2}+\varepsilon \bar{\theta}_{B}^{F B} \widehat{\theta}\right]$ with $\widehat{\theta}=p+(1-p) \bar{\theta}_{B}^{F B}$ we obtain, after computations, $\overline{\mathcal{U}}_{B}^{F B}=1+I(\varepsilon) / 2$ with $I(\varepsilon)=\bar{\theta}_{B}^{F B}\left[2 \alpha_{B}-1-p \varepsilon\left(\alpha_{A}-\right.\right.$ $\left.\left.\alpha_{B}\right)\right]$. Thus, $I^{\prime}(\varepsilon)=\partial_{\varepsilon} \bar{\theta}_{B}^{F B}\left[2 \alpha_{B}-1-\varepsilon p\left(\alpha_{A}-\alpha_{B}\right)\right]-\bar{\theta}_{B}^{F B} p\left(\alpha_{A}-\alpha_{B}\right)$. As $\left.\bar{\theta}_{B}^{F B}\right|_{p=1}=$ $\left[2 \alpha_{B}-1+\varepsilon\left(\alpha_{A}+\alpha_{B}\right)\right] /\left[2 \alpha_{B}\right]$ and $\left.\partial_{\varepsilon} \bar{\theta}_{B}^{F B}\right|_{p=1}=\left(\alpha_{A}-\alpha_{B}\right) /\left(2 \alpha_{B}\right)$ we obtain, after computations, $\left.I^{\prime}(\varepsilon)\right|_{p=1}=-\varepsilon \alpha_{A}\left(\alpha_{A}-\alpha_{B}\right) / \alpha_{B}$. Moreover, it is straightforward that $\left.I^{\prime}(\varepsilon)\right|_{p=0}=\left(2 \alpha_{B}-1\right)\left(\alpha_{A}-\alpha_{B}\right) /\left(2 \alpha_{B}\right)$. Then, $\left.I^{\prime}(\varepsilon)\right|_{p=0}>0$ and $\left.I^{\prime}(\varepsilon)\right|_{p=1}<0$ and, by continuity, $\overline{\mathcal{U}}_{B}^{F B}$ is an increasing function for sufficiently low values of $p$ but a decreasing one for sufficiently high values of $p$.

We now compare $\mathcal{U}^{F B}$ with $\mathcal{U}^{G}$ for type $A$ individuals. It is straightforward to establish that $\left.\mathcal{U}_{A}^{F B}\right|_{\varepsilon=0}=\left.\mathcal{U}_{A}^{L F}\right|_{\varepsilon=0}$. Moreover, according to Proposition $3,\left.\mathcal{U}_{A}^{G}\right|_{\varepsilon=0}<$ $\left.\mathcal{U}_{A}^{L F}\right|_{\varepsilon=0}$. Then $\left.\mathcal{U}_{A}^{F B}\right|_{\varepsilon=0}>\left.\mathcal{U}_{A}^{G}\right|_{\varepsilon=0}$. Similarly, $\left.\mathcal{U}_{A}^{G}\right|_{\varepsilon=\widetilde{\varepsilon}_{G}}=\left(1+\widetilde{\varepsilon}_{G}\right) \alpha_{A}$ whereas $\left.\mathcal{U}_{A}^{F B}\right|_{\varepsilon=\widetilde{\varepsilon}_{G}}=$ $\left[1+\widetilde{\varepsilon}_{G}\left(p+\left.(1-p) \bar{\theta}_{B}\right|_{\varepsilon=\widetilde{\varepsilon}_{G}} ^{F B}\right)\right] \alpha_{A}$. As $p+\left.(1-p) \bar{\theta}_{B}\right|_{\varepsilon=\widetilde{\varepsilon}_{G}} ^{F B}<1$ we have $\left.\mathcal{U}_{A}^{G}\right|_{\varepsilon=\widetilde{\varepsilon}_{G}}>\left.\mathcal{U}_{A}^{F B}\right|_{\varepsilon=\widetilde{\varepsilon}_{G}}$. By continuity we have established that $\mathcal{U}_{A}^{F B}>\mathcal{U}_{A}^{G}$ when $\varepsilon$ is sufficiently low whereas $\mathcal{U}_{A}^{F B}<\mathcal{U}_{A}^{G}$ for sufficiently large values of $\varepsilon$.

We finally compare $\mathcal{U}^{F B}$ with $\mathcal{U}^{G}$ for type $B$ individuals. It is straightforward to establish that $\left.\mathcal{U}_{B}^{F B}\right|_{\varepsilon=0}=\left.\mathcal{U}_{B}^{L F}\right|_{\varepsilon=0}$. Moreover, according to Proposition $4,\left.\mathcal{U}_{B}^{G}\right|_{\varepsilon=0}<$ $\left.\mathcal{U}_{B}^{L F}\right|_{\varepsilon=0}$. Hence, $\left.\mathcal{U}_{B}^{F B}\right|_{\varepsilon=0}>\left.\mathcal{U}_{B}^{G}\right|_{\varepsilon=0}$. By definition of the First Best, we have for all $\varepsilon, p \mathcal{U}_{A}^{F B}+(1-p) \mathcal{U}_{B}^{F B} \geq p \mathcal{U}_{A}^{G}+(1-p) \mathcal{U}_{B}^{G}$. As $\left.\mathcal{U}_{A}^{G}\right|_{\varepsilon=\widetilde{\varepsilon}_{G}}>\left.\mathcal{U}_{A}^{F B}\right|_{\varepsilon=\widetilde{\varepsilon}_{G}}$, we necessarily have $\left.\mathcal{U}_{B}^{G}\right|_{\varepsilon=\widetilde{\varepsilon}_{G}}>\left.\mathcal{U}_{B}^{F B}\right|_{\varepsilon=\widetilde{\varepsilon}_{G}}$. By continuity we have established that $\mathcal{U}_{B}^{F B}>\mathcal{U}_{B}^{G}$ when $\varepsilon$ is sufficiently low or sufficiently large. 